\title{
1 Prefrontal cortical activity predicts the extra-place field spiking of hippocampal place cells
}

2 Jai Y. Yu ${ }^{1 *}$ and Loren M. Frank ${ }^{2}$

3 1. Department of Psychology, Institute for Mind and Biology, Grossman Institute for

4 Neuroscience, Quantitative Biology and Human Behavior, The University of Chicago

2. Howard Hughes Medical Institute, Kavli Institute for Fundamental Neuroscience, Departments of Physiology and Psychiatry, University of California, San Francisco

* Corresponding author

\section{Abstract}

The receptive field of a neuron describes the regions of a stimulus space where the

13 neuron is consistently active. Sparse spiking outside of the receptive field is often considered to

14 be noise, rather than a reflection of information processing. Whether this characterization is

15 accurate remains unclear. We therefore contrasted the sparse, temporally isolated spiking of

16 hippocampal CA1 place cells to the consistent, temporally adjacent spiking seen within their

17 spatial receptive fields ("place fields"). We found that isolated spikes, which occur during

18 locomotion, are more strongly phase coupled to hippocampal theta oscillations than adjacent

19 spikes and, surprisingly, transiently express coherent representations of non-local spatial

20 representations. Further, prefrontal cortical activity is coordinated with, and can predict the

21 occurrence of future isolated spiking events. Rather than local noise within the hippocampus,

22 sparse, isolated place cell spiking reflects a coordinated cortical-hippocampal process consistent

23 with the generation of non-local scenario representations during active navigation.

\section{Main text}

The concept of a receptive field (Sherrington 1906, Hartline 1938, Spillmann 2014)

27 provides a fundamental model for how neural spiking can convey information about features in

28 the external environment. In the hippocampus, many cells show spatially tuned receptive fields

29 (O'Keefe and Dostrovsky 1971, O'Keefe 1976). The spiking rate of these "place cells" rises and 
then falls as an animal traverses specific locations in an environment. Locations with high spiking rates are defined as a cell’s “place fields” (O'Keefe 1976, McNaughton, Barnes et al.

32 1983), and place field-associated spiking of place cells conveys sufficient spatial information to

33 estimate the animal's location with high accuracy (Muller and Kubie 1989, Wilson and

34 McNaughton 1993, Brown, Frank et al. 1998, Zhang, Ginzburg et al. 1998).

Although the majority of place cell spiking occurs when an animal is moving within the cell's place fields, occasional spiking occurs when the animal is at locations outside the fields (O'Keefe 1976, McNaughton, Barnes et al. 1983, Muller, Kubie et al. 1987, Thompson and Best 1989). These "isolated" spiking events can occur during movement and are distinct from sparse spiking observed during Sharp Wave/Ripples (SWRs) seen during immobility (Buzsáki 2015). Importantly, isolated spikes are not locked to specific locations. As a result, standard analyses that average activity across many passes through the same location (Olton, Branch et al. 1978,

42 Hill and Best 1981, Thompson and Best 1989, Wilson and McNaughton 1993, Frank, Brown et

43 al. 2000, Jensen and Lisman 2000) effectively exclude these spikes from further consideration.

44 Whether these spikes reflect unreliable, noisy processes that merit exclusion or whether they 45 instead reflect coherent, meaningful signals (Stein, Gossen et al. 2005, Faisal, Selen et al. 2008, 46 Masquelier 2013) remains unknown.

Noise in neural networks can arise from stochastic cellular events that cause the membrane voltage to occasionally exceed the action potential threshold, even without upstream input (Stein, Gossen et al. 2005, Faisal, Selen et al. 2008). While the spatially selective inputs to a place cell raise the membrane voltage closer to the action potential threshold when an animal approaches the cell's place field (Epsztein, Brecht et al. 2011), stochastic events causing

52 occasional increases in membrane potential could result in spiking outside of a cell's place field.

53 However, previous observations indicate that at least some spiking outside of a cell's typical

54 place fields reflect mnemonic processes rather than noise. CA1 and CA3 place cells can emit

55 spikes outside of their place fields as an animal approaches choice points (Johnson and Redish

56 2007, Kay, Chung et al. 2020) and during vicarious trial and error (Johnson and Redish 2007), or

57 when an animal is travelling in the opposite direction over a location with a place field (Kay,

58 Chung et al. 2020). These events are hypothesized to reflect non-current scenarios, such as 
59 simulating possible future scenarios when a decision needs to be made (Johnson, Fenton et al.

60 2009, Kay, Chung et al. 2020).

How can we determine whether isolated spiking outside of a place cell's spatially tuned

62 receptive field reflects information processing in the hippocampal circuit as opposed to activity

63 that does not reflect information processing or noise? Spiking due to stochastic cellular events is

64 expected to be local to individual neurons. By contrast, spiking associated with information

65 processing would be expected to co-vary in a consistent manner across neurons in both local and

66 distributed networks (Masquelier 2013). Thus, if spiking outside of the classical place field

67 conveys information, we would expect it to (1) be coordinated across multiple hippocampal

68 neurons, (2) contain coherent spatial information and (3) be coordinated with activity outside the

69 hippocampus.

70 We therefore examined spiking both within the hippocampus and across the hippocampus

71 and prefrontal cortex (PFC), focusing on activity during movement. The PFC is anatomically

72 connected to the hippocampus through both direct and indirect projections (Swanson and Cowan

73 1977, Swanson 1981, Jay, Glowinski et al. 1989), and coordinated activity across these networks

74 reflects their engagement during memory processing (Schacter, Addis et al. 2007, Preston and

75 Eichenbaum 2013, Eichenbaum 2017). For example, network level coherence between prefrontal

76 cortex and hippocampus increases during periods when memory retrieval occurs (Hyman, Zilli et

77 al. 2005, Jones and Wilson 2005, Benchenane, Peyrache et al. 2010, Sigurdsson, Stark et al.

78 2010, Place, Farovik et al. 2016, Guise and Shapiro 2017, Myroshnychenko, Seamans et al.

79 2017, Zielinski, Shin et al. 2019). Whether PFC activity differs systematically at the time of

80 isolated spiking in the hippocampus remains unknown.

81 Our examination of isolated spiking of place cells revealed that these events reflect the

82 coherent activation of hippocampal representations of physically distant locations, and that these

83 events are coordinated with ongoing activity in the PFC. These findings suggest that isolated

84 spikes are a signature of distributed and coherent information processing in the brain.

\section{Results}


In order to understand the extent of isolated spiking during active behavior and to identify

87 a potential function of this activity, we took an unbiased approach where we surveyed CA1 place cell spiking across all movement periods (animal speed $>2 \mathrm{~cm} / \mathrm{s}$ ) as animals performed a spatial navigation task (Yu, Kay et al. 2017, Yu, Liu et al. 2018) (Figure 1A-B, Figure S1). In the hippocampus the temporal structure of spiking during locomotion is strongly influenced by the endogenous $\sim 8 \mathrm{~Hz}$ theta rhythm (O'Keefe and Recce 1993), and bouts of higher rate spiking corresponding to place field traversals spanned multiple, adjacent cycles of theta (Fig. 1C). As expected, we also observed isolated spikes where a neuron would be silent for many theta cycles, emit a small number of spikes on a single theta cycle, and then return to being silent (Fig. 1D) (Olton, Branch et al. 1978, Hill and Best 1981, Thompson and Best 1989, Johnson and Redish 2007).

The standard approach to defining place field spiking relies on averaging spiking rates across many traversals of a location. This average provides a useful experimental summary of spiking, but information averaged across traversals is not directly available to downstream neurons. We therefore we used a criterion to distinguish between "adjacent" and "isolated" spiking based on the local temporal organization of spiking. Specifically, given the importance of theta in organizing hippocampal activity (Vanderwolf 1969, O'Keefe and Recce 1993,

103 Buzsaki 2002), we calculated the interval between neighboring theta cycles with spiking (in cycles, mean of nearest three) (Fig. 1E, Fig. S2). As expected, the majority of spike-containing theta cycles are near another spike-containing cycle. The remaining spike-containing theta cycles

106 are separated from neighboring spike-containing cycles by up to hundreds of cycles, reflecting 107 their temporal isolation. When plotted on a log scale, the underlying distribution was bimodal, 108 and based on this distribution we chose a threshold of 8 cycles of mean separation to each theta 109 cycle with spiking to define "adjacent" or "isolated" activity ( $\mathrm{n}=301$ cells, Fig. 1F). This 110 separation captured intuitive notions of within- and extra-place field activity: adjacent activity 111 was spatially concentrated, as expected from place-field spiking (Fig. 1E); isolated activity was 112 spatially sparse and lack the high spiking rates observed for place field activity (Fig. 1G). We 113 also verified that isolated activity spikes, although sparsely emitted, were very unlikely to be 114 spike clustering errors (Fig. S3). 
One previously described form of extra-field spiking occurred around choice points and coincides with overt deliberation behavior (Johnson and Redish 2007). Isolated activity, in contrast, was not more frequent around choice point locations (Fig. S4A), nor were there differences in the speed (Fig. S4B) or angular velocity (Fig. S4C) of the animal at times of isolated as compared to adjacent spiking. Thus, isolated spiking is not restricted to specific active behavioral states or locations, such as path choice points. Isolated spiking was also not associated with SWRs, which are transient network oscillations observed in the local field potential (LFP) predominantly found when the animal is moving slowly or is immobile (Buzsáki 2015). We excluded SWRs from our analyses (see methods) and also independently confirmed the isolated spiking events did not occur during SWRs by comparing the hippocampal LFP associated with excluded spiking (low speed periods and SWRs) with the LFP associated with isolated spiking. The LFP associated with excluded spiking showed a network spectral signature consistent with SWRs (Fig. S5A left column, B), with power in the slow gamma $(\sim 30 \mathrm{~Hz})$ and ripple frequencies $(\sim 150-250 \mathrm{~Hz})$. In contrast, the LFP associated with isolated spiking shows a different network spectral signature, with power in the theta band (Buzsaki, Leung et al. 1983) (Fig. S5A center column, B). Indeed, the network spectral signature of isolated spiking is very similarity to the LFP associated with adjacent spiking, and even has slightly higher theta power (Fig. S5A right column, B).

Isolated spiking was also highly concentrated within each theta cycle, a potential signature of an information containing signal (Masquelier 2013). Place field-associated spiking displays strong phase-coupling to the hippocampal theta rhythm, where the maximum probability of spiking occurs in earlier phases near the trough of theta (Buzsaki, Leung et al. 1983, O'Keefe and Recce 1993). By contrast, the entrainment of isolated spiking preferentially occurred in the late phases of theta (Fig. 2A-B). Isolated spiking was also more tightly phase locked to theta compared with adjacent spiking (Fig. 2C). local representations, including to be visited locations or locations previously visited (Skaggs,

142 McNaughton et al. 1996, Redish 2016, Kay, Chung et al. 2020). Could isolated spiking be a 143 signature of a coherent non-local representation? If so, then we would expect that pairs of 144 neurons that are co-active during periods of adjacent spiking (e.g. cells likely to have 
145

146

147

148

149

150

151

152

153

154

155

156

157

158

159

160

161

162

163

164

165

166

167

168

169

170

171

172

173

overlapping place fields) would also be co-active within a theta cycle containing isolated spiking events. We examined this possibility by using an approach that has been used to demonstrate reactivation of non-local spatial representations during SWRs, where a pair of place cells is more likely to spike together if their place fields overlap (Karlsson and Frank 2009, Singer and Frank 2009) (Fig. 3A). First, we calculate the likelihood of co-spiking for a pair of place cells that had isolated spiking within the same theta cycle. We then quantified the overlap in their adjacent spiking activity.

We found that cells that fired together during periods of adjacent spiking were also more likely to fire together during isolated spiking events. Across the population, lower lags in spiking during adjacent activity were correlated with greater co-spiking during isolated events (Fig. 3B, $\left.\mathrm{R}=-0.28, \mathrm{R}^{2}=0.077, \mathrm{p}=6.40 \times 10^{-9}\right)$. These findings support the notion that isolated spikes reflect the structured and coherent activation of a remote spatial representation.

As a further test of the hypothesis that isolated spiking contains information, we asked whether these spiking events in the hippocampus marked the time of specific activity patterns in PFC. Given the anatomical and functional connectivity between these regions, evidence of coordination between hippocampus and PFC would argue that these events are not the result of local noise in the hippocampus but instead reflect coherent and structured activity across brain regions. An example of such hippocampal-cortical engagement occurs during SWRs, where hippocampal reactivation is accompanied by the coordinated reactivation of cortical representations (Remondes and Wilson 2015, Jadhav, Rothschild et al. 2016, Wang and Ikemoto 2016, Rothschild, Eban et al. 2017, Yu, Kay et al. 2017, Yu, Liu et al. 2018). If such coordination is seen around the times of isolated spikes, we should be able to identify PFC neurons that spike differently around times of isolated activity in the hippocampus than at comparable periods where isolated spiking was not observed.

We first selected theta cycles with isolated spiking for a given CA1 cell. Next, we found matching theta cycles from other times when the animal was moving through the same locations in the same direction at a similar speed, but where the CA1 cell was not active (e.g. did not have isolated spiking) (Fig. S6). This was possible because, in our task, the animal traversed the a given location multiple times, providing a pool of theta cycles, of which only a subset contained 
174 isolated spiking. Importantly, none of the matching cycles contained adjacent spiking,

175 confirming that the isolated spiking events were not simply events on the edge of a place field.

176 We then compared the spiking of simultaneously recorded PFC neurons between cycles with

177 isolated activity and these matched control cycles (Fig. 4A). We note that theta coordinates

178 activity in hippocampal-cortical networks (Hyman, Zilli et al. 2005, Jones and Wilson 2005),

179 allowing us to continue to use theta cycles as the temporal reference to relate activity across

180 structures.

We found PFC cells whose spiking rate differed depending on whether or not there was an associated period of isolated spiking for a given CA1 cell (Fig. 4B). We expect that only a small fraction of PFC cells would show a significant difference in spiking relative to the isolated spiking of a given CA1 cell, but nonetheless, across the population ( $\mathrm{n}=2798$ PFC-CA1 cell pairs), the difference in PFC firing rates between isolated and matched control periods was significantly larger than the permutation control (Fig. 4C). This difference indicates coordination between CA1 and prefrontal cortex around the time of CA1 isolated activity. Interestingly, this coordination was not limited to the specific isolated theta cycle: the difference remained significant even in a window of 4-8 theta cycles before the isolated spiking event, indicating that PFC activity could play a causal role in driving isolated spiking events in the hippocampus.

Under this hypothesis, the ensemble activity of PFC neurons should predict the future occurrence of hippocampal isolated activity (Fig. 5A). To test that prediction for a given CA1 cell, we used the spiking activity from all simultaneously recorded PFC ensembles (Median $\mathrm{n}=20, \mathrm{IQR}=8 \mathrm{PFC}$ cells per CA1 cell) to build cross-validated Generalized Linear Models with elastic net regularization. We compared the ability of the models to predict the occurrence of isolated activity related to a permutation control (see Methods, Fig. S7). We then carried out that analysis for all CA1 cells $(\mathrm{n}=158)$ with isolated spiking. above chance levels, even in a window 4-8 theta cycles before the isolated spiking (Fig. 5B). We

200 also asked whether there was any evidence consistent with isolated spiking in CA1 influencing 201 subsequent PFC activity (Fig. 5C). We found that the coordination between hippocampus and prefrontal cortex persists after the occurrence of isolated activity but is weaker compared to 
203

204

205

206

207

208

209

210

211

212

213

214

215

216

217

218

219

220

221

222

223

224

225

226

227

228

229

230

231

intervals immediately before and during cycles with isolated activity (Fig. 5D). We also noted the average prediction gains were small in magnitude, which has previously been observed for prediction gains relating auditory and hippocampal activity around the times of SWRs (Rothschild, Eban et al. 2017). This is not surprising given the relatively small numbers of simultaneously recorded PFC cells that were available to predict the activity of any given CA1 unit. We can therefore regard these cross-validated predictions as lower bounds on the actual values that would be obtained if it were possible to sample the entire PFC population. Indeed, examining the values for individual PFC ensemble - CA1 models revealed a number of cases with prediction gains between 2.5 and 5\% (Fig. S7). Thus, our results demonstrate that information expressed by prefrontal and hippocampal cell populations is coordinated around the time of isolated activity.

Importantly, the predictive PFC activity patterns were specific for individual CA1 cells. We examined the correlation between $\beta$-coefficients of PFC predictors across predictive models. If the spiking of specific PFC cells was strongly predictive of isolated spiking of a particular CA1 cell but not of other CA1 cells, this $\beta$-coefficient correlation should be low, indicating that a given PFC cell would predict the spiking in one model (e.g. one CA1 cell) but not another. By contrast, if a subset of PFC cells consistently predicted isolated spiking across CA1 cells, then these correlations would be high, as the same PFC cells would show similarly $\beta$-coefficients across models.

We found that the mean correlation coefficient was not significantly different from 0 (Median $=-0.021, \mathrm{IQR}=0.16$, Wilcoxon rank sum test $\mathrm{p}=0.431$ ). This indicates that the PFC ensembles predicting the occurrence of isolated activity for different CA1 cells are distinct, and argues for specificity in PFC-CA1 coordination around the occurrence of isolated activity.

\section{Discussion}

We examined spiking outside of a place cell's place field relative to local hippocampal network activity and to activity in prefrontal cortex. We found that this isolated spiking preferentially occurs during the late phase of theta oscillations, recapitulates coherent spatial representations, and is coordinated with prefrontal cortical activity. Our findings argue that seemingly spontaneous and sparse activity, previously considered as noise in the hippocampus, 
232

233

234

235

236

237

238

239

240

241

242

243

244

245

246

247

248

249

250

251

252

253

254

255

256

257

258

259

260

are actually precisely timed spikes that reflect coordinated activity both within the hippocampus and across the hippocampal-prefrontal networks.

We found evidence for CA1 isolated spiking reflecting structured information processing rather than noise within the hippocampal network at both the single cell and pairwise level of analysis. At the single cell level, isolated CA1 spiking is highly concentrated during in the late phases of theta, pointing to the segregation of current versus non-current scenarios between the early and late phase of theta respectively (Sanders, Renno-Costa et al. 2015). This is in line with previously described place cell spiking associated with non-local representations including possible future locations, travel in the non-current direction (Wang, Foster et al. 2020), and activity on non-preferred trajectories (Kay, Chung et al. 2020), all of which are seen preferentially during the late phases of theta. Our results also extend previous findings of nonlocal spiking associated with vicarious trial and error behavior seen near choice points or at the edges of place fields (Johnson and Redish 2007, Redish 2016). We found that isolated spikes occur throughout the environment and are not concentrated at the edges of place fields or near choice points. These spikes also occurred in association with high movement speeds. Pairwise analyses further demonstrated that isolated spikes are coordinated across hippocampal neurons: cells that fired together during adjacent spiking periods were also more likely to fire together within an isolated spiking event. This is consistent with a brief, coherent activation of a remote spatial representation, indicating that these events could support deliberative processes associated with the evaluation of distant physical locations.

Our analysis of isolated CA1 place cell spiking relative to PFC activity provided additional evidence that these events could support deliberative processes. At the single pair level, we identified individual PFC neurons that spiked differently in association with an isolated CA1 spiking event as compared to periods matched for location, direction of movement, and speed. At the ensemble level we found that these differences were significant not only during the theta cycle associated with isolated CA1 spiking, but also 4-8 cycles before the isolated spiking event. Moreover, ensemble PFC activity could predict the occurrence of a theta cycle with isolated CA1 spiking, and these predictions remained significant for PFC activity occurring 4-8 cycles before the isolated spiking. These predictions were also specific: a particular set of PFC 
261

262

263

264

265

266

267

268

269

270

271

272

273

274

275

276

277

278

279

280

281

282

283

284

285

286

287

288

289

cells were strong predictors of a given CA1's cells isolated spiking, while a different set of PFC cells might predict isolated spiking in a different CA1 cell.

These findings indicate that isolated spiking events in CA1 are very unlikely to be the product of local, stochastic fluctuations in the hippocampus. Instead, our findings indicate the presence of a slow $(\sim 1 \mathrm{~s})$ change in PFC activity that could trigger an isolated spiking event in CA1. Interestingly, the changes in rate in PFC and the strength of the PFC-CA1 coupling is greatest during the cycle with isolated spiking but remains greater than expected by chance well after the CA1 spiking, suggesting the possibility that the CA1 spiking drives a subsequent change in PFC activity. Thus, our findings point to a potential cortical-hippocampal-cortical of information flow, conceptually similar to the cortical-hippocampal-cortical information flow seen around SWRs during sleep (Rothschild, Eban et al. 2017).

Our results also suggest that information exchange between cortex and hippocampus may occur frequently during active behavior. This extends previous findings of hippocampalprefrontal coupling from imaging (Squire, Ojemann et al. 1992, Buckner, Petersen et al. 1995, Schacter, Alpert et al. 1996, Polyn, Natu et al. 2005, St Jacques, Kragel et al. 2011, Rugg and Vilberg 2013, Schedlbauer and Ekstrom 2019) and neural recording experiments (Tomita, Ohbayashi et al. 1999, Kyd and Bilkey 2003, Hyman, Zilli et al. 2005, Jones and Wilson 2005, Benchenane, Peyrache et al. 2010, Sigurdsson, Stark et al. 2010, Hok, Chah et al. 2013, Place, Farovik et al. 2016, Guise and Shapiro 2017, Myroshnychenko, Seamans et al. 2017, Zielinski, Shin et al. 2019) on the role of prefrontal cortex in modulating both cortical and subcortical structures during mnemonic processes (Tomita, Ohbayashi et al. 1999, Simons and Spiers 2003, St Jacques, Kragel et al. 2011, Eichenbaum 2017). Our results also complement findings demonstrating coherent spiking activity patterns across hippocampus and PFC in the context of both SWRs and locomotion-associated spiking (Jadhav, Rothschild et al. 2016, Shin, Tang et al. 2019, Zielinski, Shin et al. 2019).

Interestingly, the communication latency between PFC and HP is hypothesized to be $\sim 26-28 \mathrm{~ms}$, or approximately $1 / 4$ of a theta cycle (Place, Farovik et al. 2016). We were therefore surprised to find PFC spiking can predict whether isolated spiking will occur up to 4-8 theta cycles, or approximately $500 \mathrm{~ms}-1 \mathrm{~s}$, later, an interval much longer than what is needed for 
290 direct information transfer. Although the channel for communication between PFC and

291 hippocampus may have a short latency, our results suggest the expression of isolated

292 hippocampal spiking likely involves coordinated activity between these regions (Squire,

293 Ojemann et al. 1992, Buckner, Petersen et al. 1995, Schacter, Alpert et al. 1996) that evolves

294 over time (Polyn, Natu et al. 2005, Rugg and Vilberg 2013, Schedlbauer and Ekstrom 2019).

295 This is consistent with human imaging studies that show cortical activity change can precede

296 memory recall on the order of seconds (Polyn, Natu et al. 2005). This long duration may in part

297 be explained by the timescale of cortical processing where spiking time constants are $>100 \mathrm{~ms}$

298 (Murray, Bernacchia et al. 2014). The long timescale may reflect additional intracortical

299 communication necessary to integrate information across multiple theta cycles, which eventually

300 triggers the expression of hippocampal representations.

We hypothesize that these exchanges may serve to modulate ongoing hippocampal-

cortical network representations corresponding to current experience with internally generated representations corresponding to non-current scenarios. Additionally, we hypothesize that through this mode of communication, PFC could drive the expression of non-current scenario representations from memory in the hippocampus, which in turn, feeds back to cortical regions as a part of an evaluation loop (Yu and Frank 2015). This coordination could be involved in covert evaluation of potential trajectories or goal locations for decision making in the future. The cortical drive could potentially underly previously reported extra-place field spiking and noncurrent spatial representations in the hippocampus associated with approach to a choice point

310 (Kay, Chung et al. 2020), during vicarious trial and error (Johnson and Redish 2007), and 311 spiking during travel in the non-preferred direction of place fields (Kay, Chung et al. 2020).

Both cortical and hippocampal spiking patterns display noise-like variation, even when

313 the animal performs repeated tasks or actions (Tolhurst, Movshon et al. 1983, Fenton, 1998

314 \#2585, Shadlen and Newsome 1998). However, cortical discharge can also be highly

315 reproducible given a consistent input (Mainen and Sejnowski 1995), and behaviors can reflect a

316 degree of accuracy consistent with very low levels of noise in the brain (Osborne, Lisberger et al.

317 2005). In the context of signal versus noise, our findings indicate that the sparse spiking of

318 hippocampal place cells is better understood as a signal that express non-current representations

319 consistent with alternative scenarios. These transient injections of non-current representations 
320 could signal processes that update ongoing hippocampal representations. Thus, the hippocampal

321 place cell spiking during active behavior may dynamically reflect both externally driven and

322 internally generated, non-current representations, which we hypothesize can collectively guide

323 ongoing behavior.

324

325 Author contributions

326 J.Y.Y and L.M.F. designed the analysis and wrote the manuscript. J.Y.Y analyzed the data.

\section{Acknowledgements}

328 We thank A. Comrie, A. Gillespie, J. Guidera, A. Joshi and K. H. Lee for comments on the

329 manuscript, and D. Liu, A. Loback and I. Grossrubatscher for assisting in the collection of the

330 original data. This work was supported by a Jane Coffin Childs Memorial Fund for Biomedical

331 Research postdoctoral fellowship (J.Y.Y.), the Howard Hughes Medical Institute, and University

332 of California Office of the President Lab Fees Award \#LF-12-237680 (L.M.F.).

\section{Data Availability}

335 Data used for this manuscript can be accessed at: https://crcns.org/data-sets/hc/hc-13/about-hc-13

337 Code Availability

338 Statistical analysis was performed using standard MATLAB, Scipy, Numpy modules listed in the 339 methods and text. Figures were generated using MATLAB and Matplotlib. The code is available 340 upon request. 
bioRxiv preprint doi: https://doi.org/10.1101/2020.11.23.395012; this version posted November 24, 2020. The copyright holder for this preprint (which was not certified by peer review) is the author/funder, who has granted bioRxiv a license to display the preprint in perpetuity. It is made available under aCC-BY-NC-ND 4.0 International license.

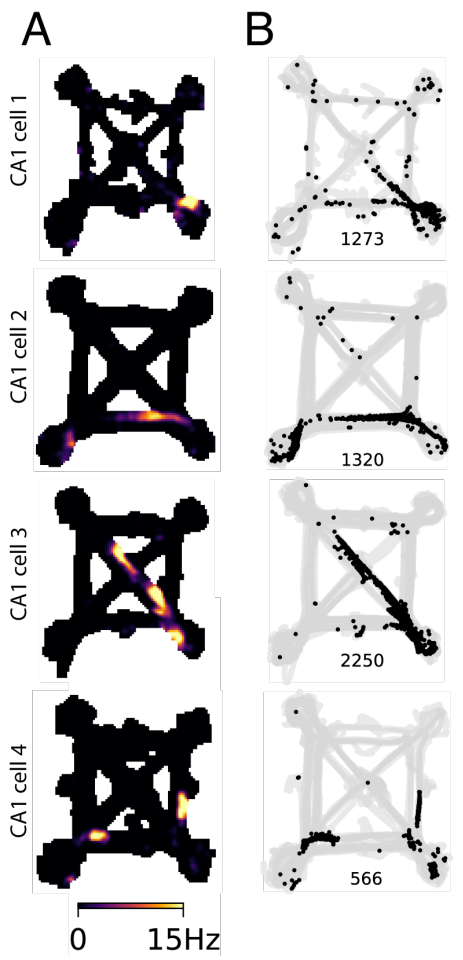

C

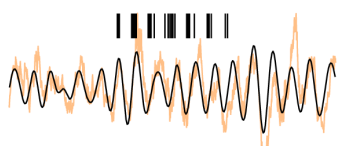

\section{| | | || || | |||| || || ||||| | |}

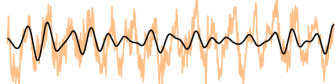

| || || | | | |

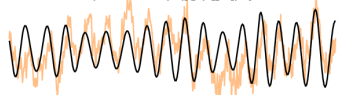

| || | |||| || || | |

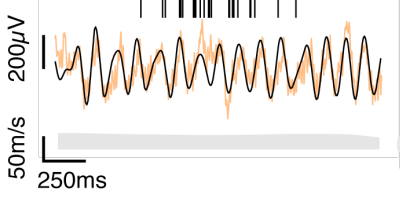

$-0-400 \mathrm{~Hz}$

$-4-12 \mathrm{~Hz}$

F

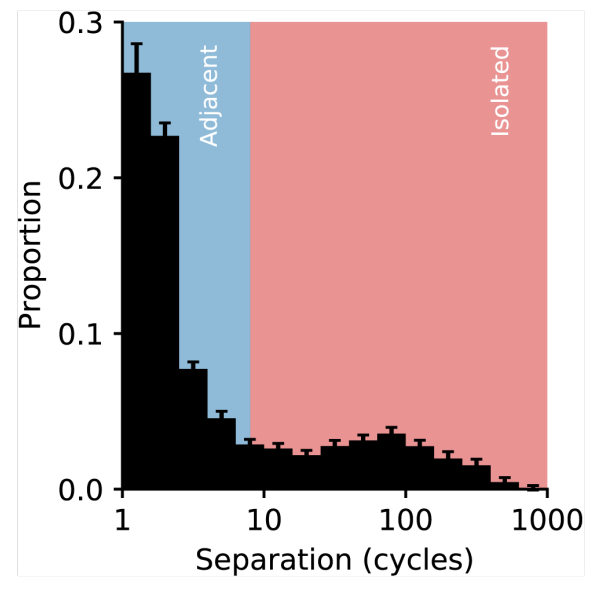

Speed
D

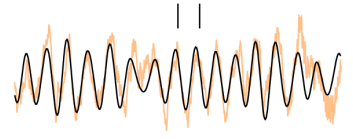

whmm
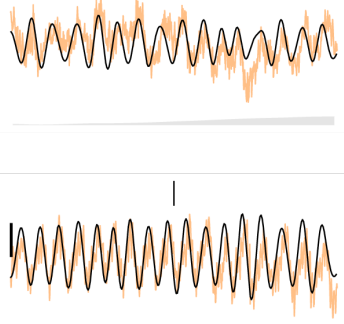

E

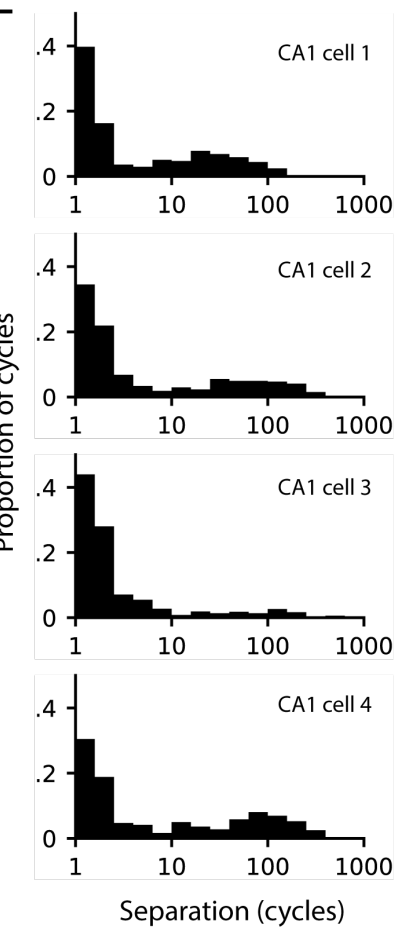

G

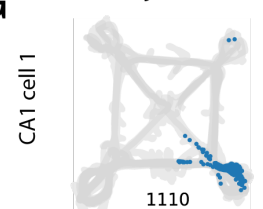

$\frac{\mathfrak{s}}{\bar{d}}$
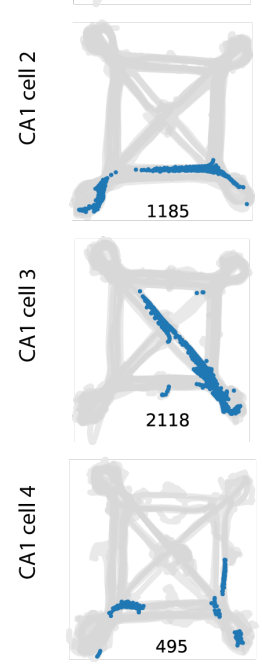

Isolated
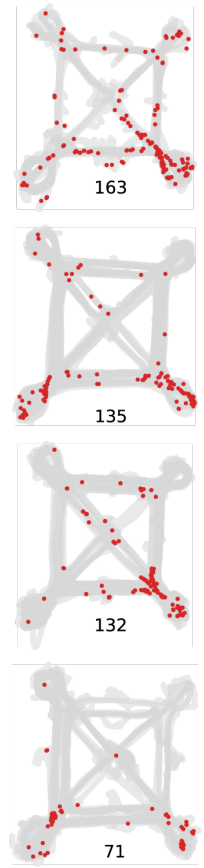
345 A. Occupancy normalized spiking rate maps for spiking activity during active movement

346 (animal speed $>2 \mathrm{~cm} / \mathrm{s}$ ) across behavior sessions for each day for four example CA1 cells.

347 B. Location of spiking (black dots) and animal trajectory (gray) for occupancy maps in A. Spike $348 \quad$ count shown below each panel.

349 C. Spike raster and corresponding location for a bout of spiking activity over adjacent theta

350 cycles. Raw (orange) and theta frequency filtered (black) local field potential are shown

351 below the spike raster. The corresponding location on the maze for the bout is shown on the 352 right.

353 D. Spike raster and corresponding location for spiking isolated from other spiking activity. The 354 corresponding location on the maze for the bout is shown on the right.

355 E. Distribution of mean separation between theta cycles with spiking. Separation is defined as 356 the mean cycle count to 3 nearest neighbor cycles with spiking.

357 F. Population distribution of mean separation between a theta cycles ( $n=301$ cells).

358 G. Location of spiking classified as adjacent activity ( $<8$ mean cycles of separation) or isolated 359 activity ( $\geq$ mean 8 cycles of separation) for the 4 example cells in A. Spike count shown 360 below each panel. 
A

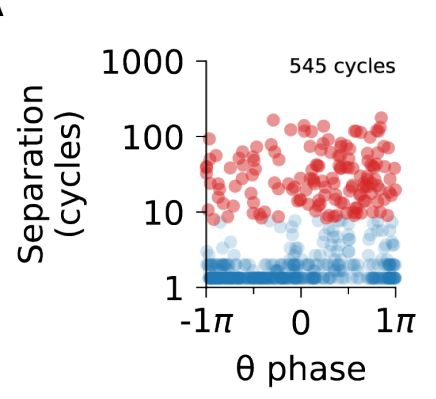

CA1 cell 1

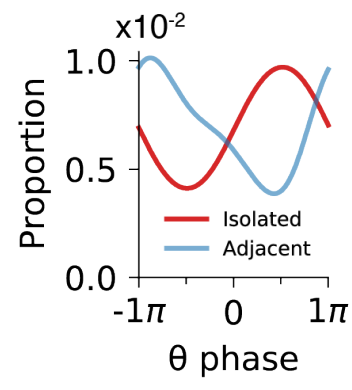

CA1 cell 3
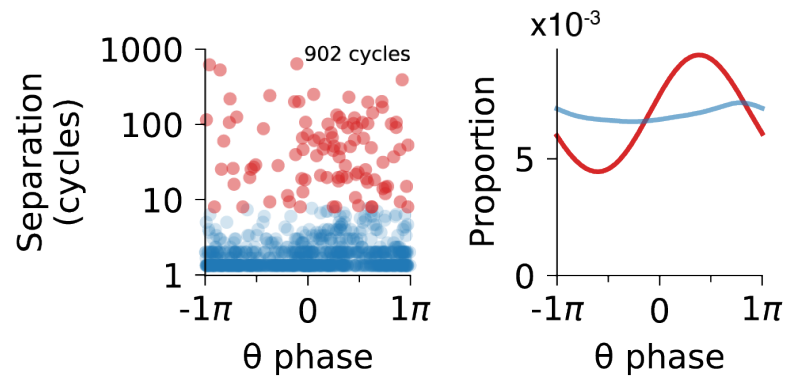

$\mathrm{B}$

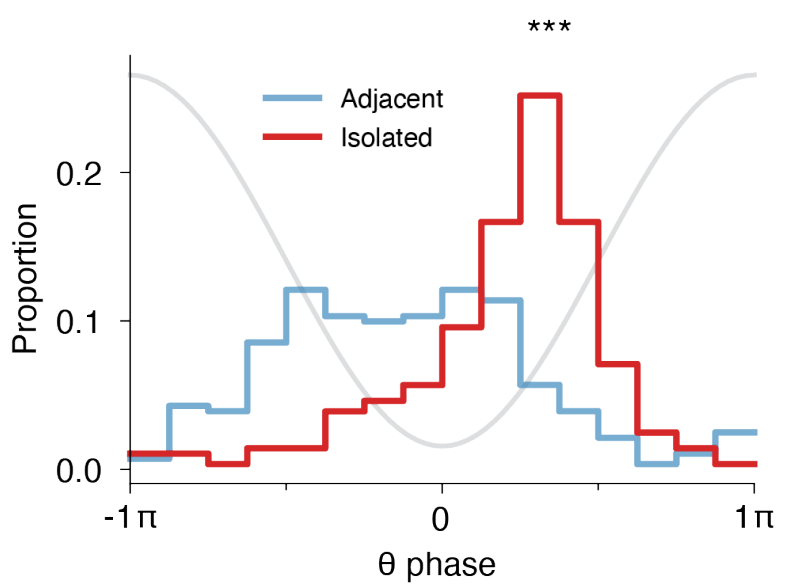

CA1 cell 2
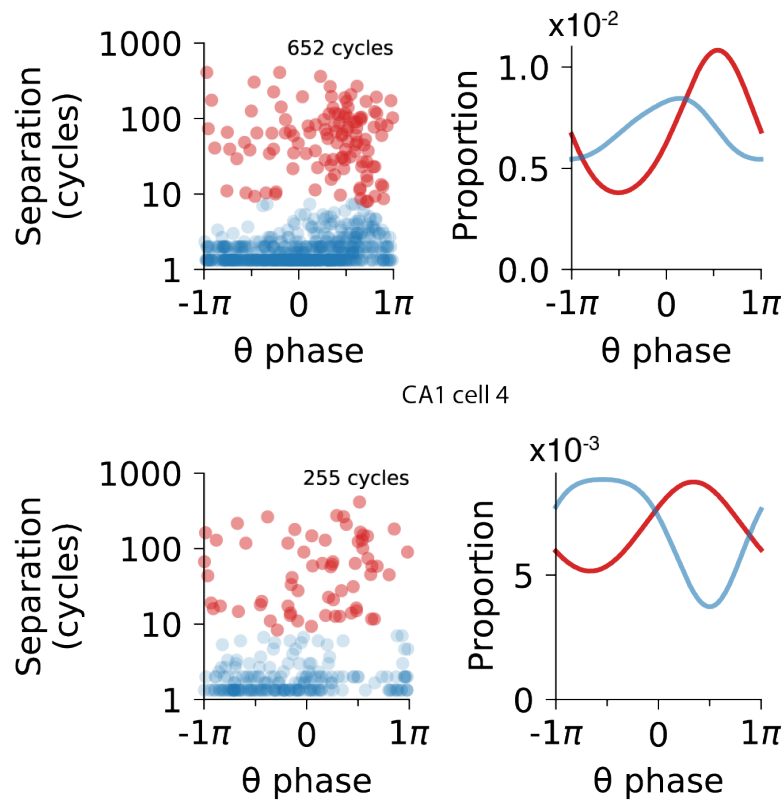

C

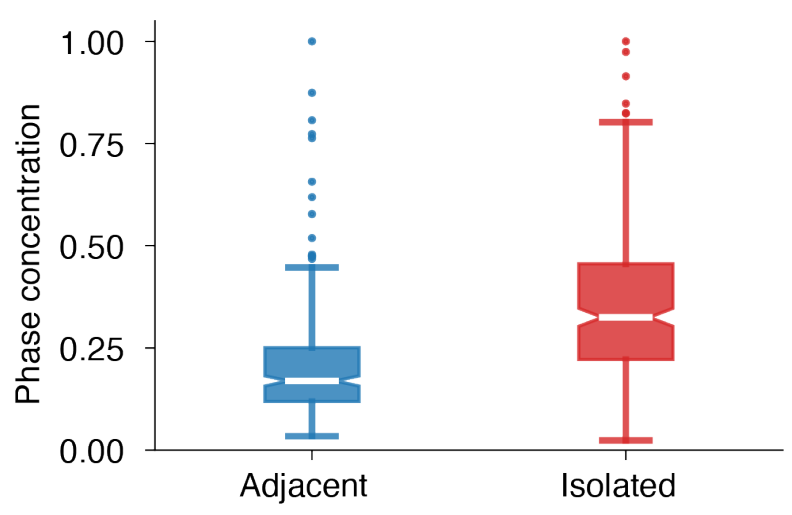

Figure 2. Isolated and adjacent spiking activity show distinct phase locking to hippocampal theta oscillation.

A. Separation versus mean spike theta phase preference for each theta cycle with spiking. A separation threshold of 8 cycles between isolated and adjacent is based on Fig. 1. Histogram shows the mean spiking phase for each theta cycle. Examples correspond to the 4 cells from Fig. 1. Circular median test between the isolated and adjacent distributions: top left $p=1.4 \times 10^{-5}$; top right $\mathrm{p}=8.9 \times 10^{-8}$; bottom left $\mathrm{p}=2.4 \times 10^{-4}$; bottom right $\mathrm{p}=5.3 \times 10^{-2}$.

B. Mean theta phase preference distribution for adjacent and isolated spiking for the CA1 place cell population ( $n=279$ cells). Gray line illustrates theta phase. Circular median test: $p=0$. 
bioRxiv preprint doi: https://doi.org/10.1101/2020.11.23.395012; this version posted November 24, 2020. The copyright holder for this preprint (which was not certified by peer review) is the author/funder, who has granted bioRxiv a license to display the preprint in perpetuity. It is made available under aCC-BY-NC-ND 4.0 International license.

372 C. Mean theta phase concentration distribution for adjacent and isolated spiking for the CA1

373 place cell population ( $n=279$ cells). Wilcoxon rank-sum test: $\mathrm{p}=4.4 \times 10^{-26}$

374

375

376

377

378 
A

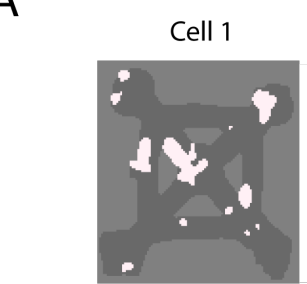

Cell 2
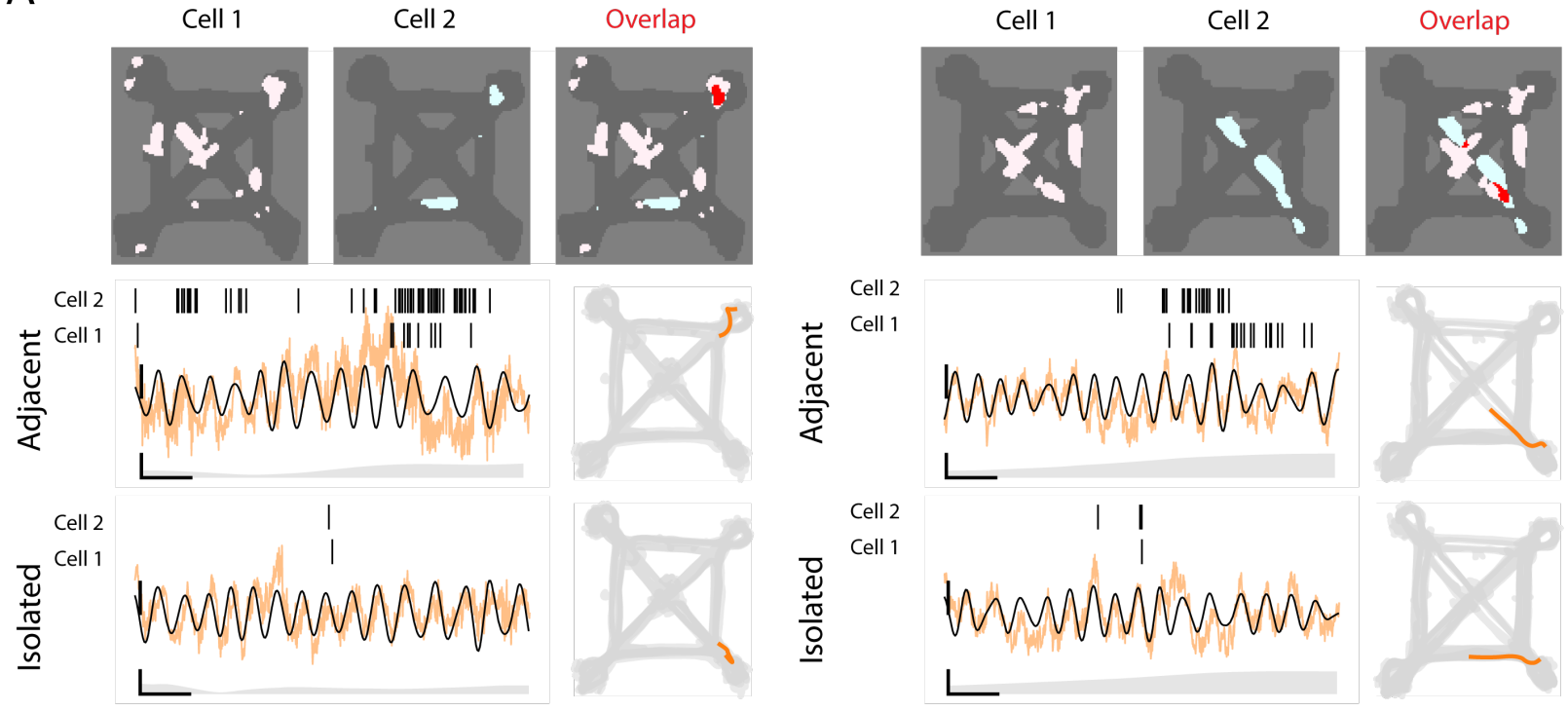

Overlap

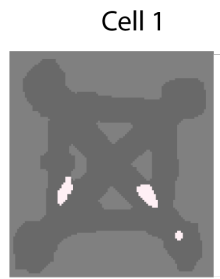

Cell 2

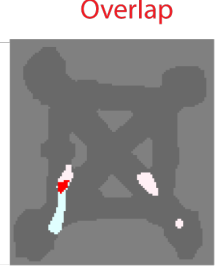

B
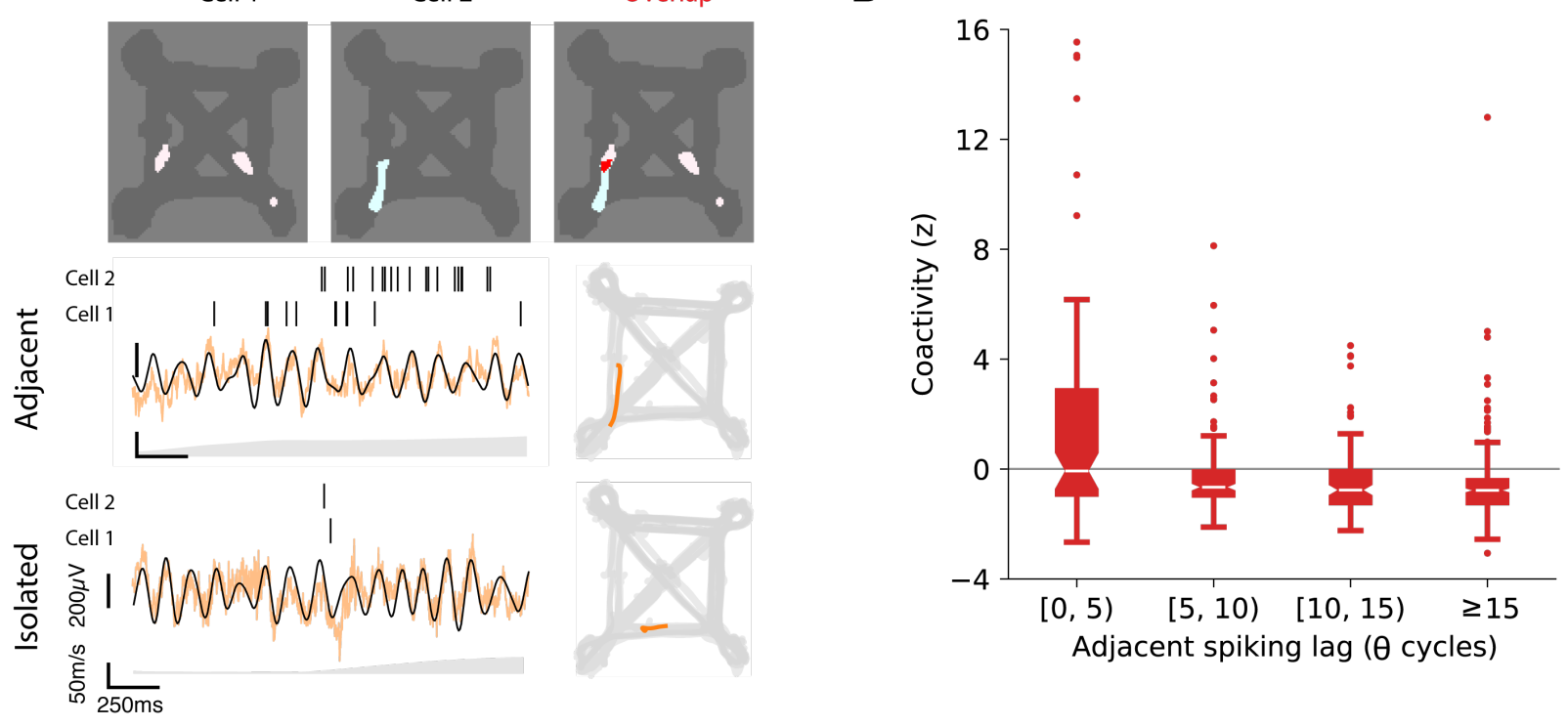

$-0-400 \mathrm{~Hz}$ - $4-12 \mathrm{~Hz}$

Speed

Figure 3. Reactivation of spatiotemporal place field activity relationships during theta

A. Three pairs of CA1 cells with overlapping adjacent activity. The place fields (occupancy normalize spiking rate $>5 \mathrm{~Hz}$ ) for each cell as well as their spatial overlap are shown. frequency band filtered LFP as well as their corresponding locations. 
bioRxiv preprint doi: https://doi.org/10.1101/2020.11.23.395012; this version posted November 24,2020 . The copyright holder for this preprint (which was not certified by peer review) is the author/funder, who has granted bioRxiv a license to display the preprint in perpetuity. It is made available under aCC-BY-NC-ND 4.0 International license.

B. Normalized coactivity (z) for CA1 cell pairs during theta cycles with isolated activity ( $\mathrm{n}=425$

387 pairs) grouped by the mean separation in time (mean lag) between their adjacent activity

388 $\left(\mathrm{R}=-0.28, \mathrm{R}^{2}=0.077, \mathrm{p}=6.40 \times 10^{-9}\right)$. 
bioRxiv preprint doi: https://doi.org/10.1101/2020.1123.395012; this version posted November 24, 2020. The copyright holder for this preprint (which was not certified by peer review) is the author/funder, who has granted bioRxiv a license to display the preprint in perpetuity. It is made available under aCC-BY-NC-ND 4.0 International license.

A

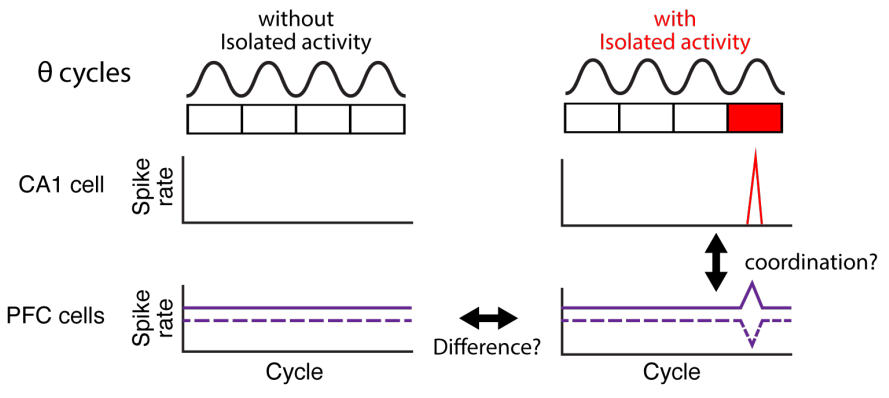

B

Cell pair 1
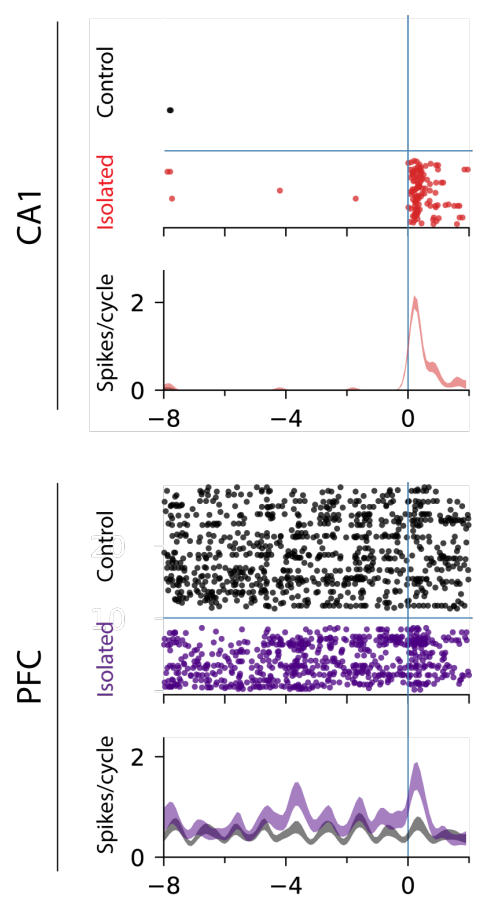

Cell pair 2

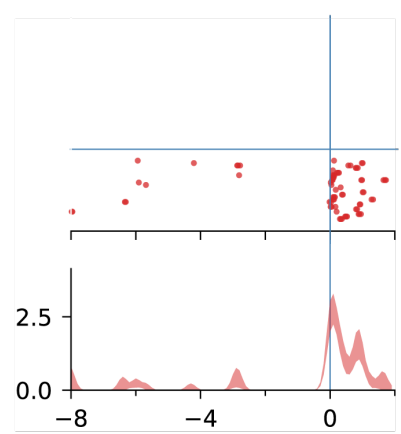

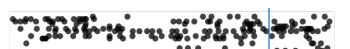
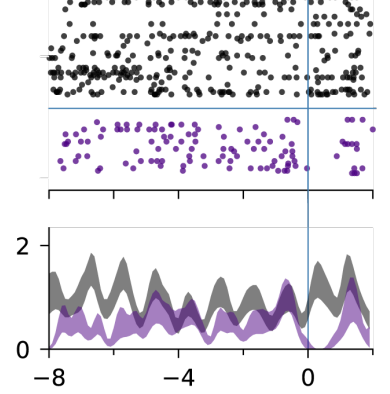

Cell pair 3
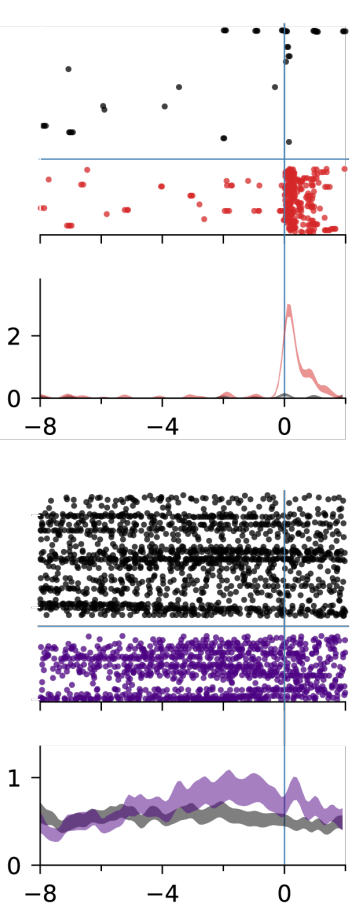

5
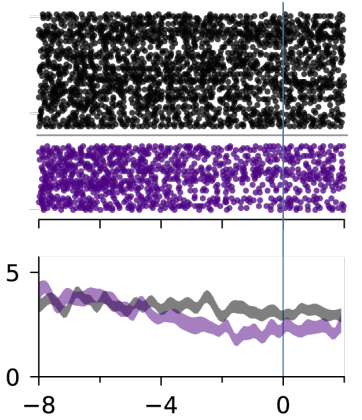

C

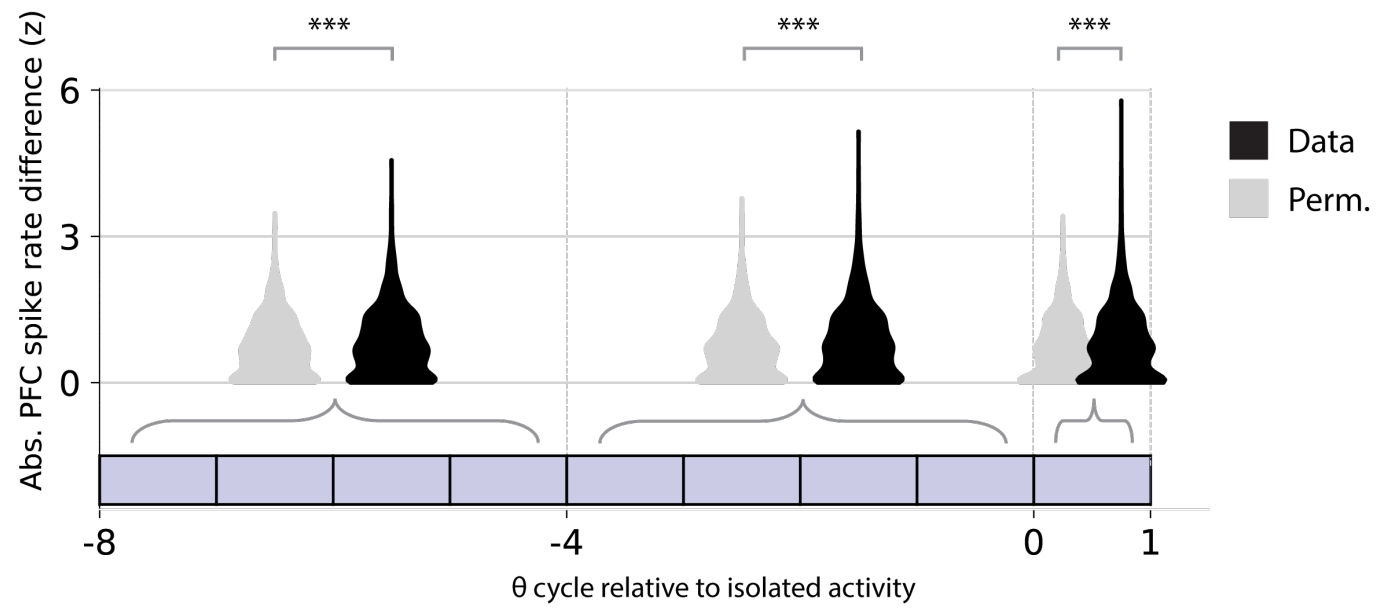




\section{Figure 4. PFC activity is coordinated with hippocampal isolated activity.}

392 A. Schematic illustrating potential CA1 and PFC activity around the time of isolated spiking. Changes in PFC spiking around the time of CA1 isolated spiking may reflect coordination

394 between the two regions.

395 B. Example spike raster and spiking rate (Mean \pm SEM) for pairs of co-recorded hippocampus CA1 and PFC cells. Each raster shows spiking aligned to isolated hippocampal activity (cycle 0) and matched control trials. Spiking is plotted relative to the cycles of the hippocampal theta rhythm. For CA1 cells, red indicates spikes and spiking rate for intervals with isolated spiking at cycle 0 . Black indicates control intervals without isolated spiking at cycle 0. For PFC cells, purple indicates spikes and spiking rate for intervals with isolated spiking at cycle 0 . Black indicates control intervals without isolated spiking at cycle 0 .

C. Violin plots and quantification of spike rate differences between control and actual intervals for PFC-CA1 cell pairs $(\mathrm{n}=2798)$ in time windows relative to CA1 isolated activity. Rate difference, original data (black) and permuted (gray), is expressed the z-score of the absolute observed difference relative to its own permuted distribution. The Wilcoxon signed-rank test $(* * * p<0.001)$ was used to compare the original and permuted groups: $p=4.7 \times 10^{-8}, 3.6 \times 10^{-12}$ and $2.2 \times 10^{-10}$ for each group respectively. 
A

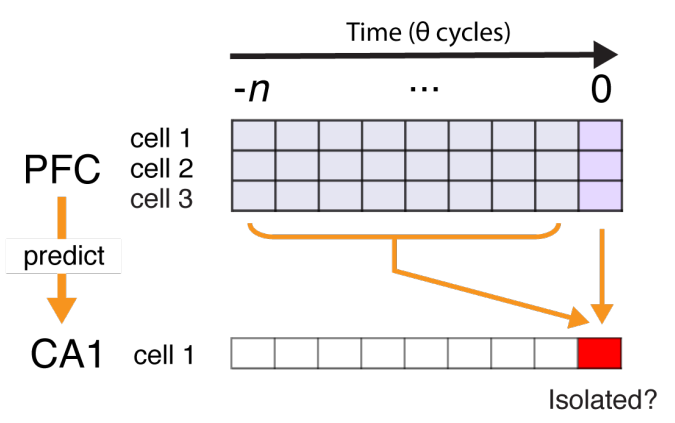

B

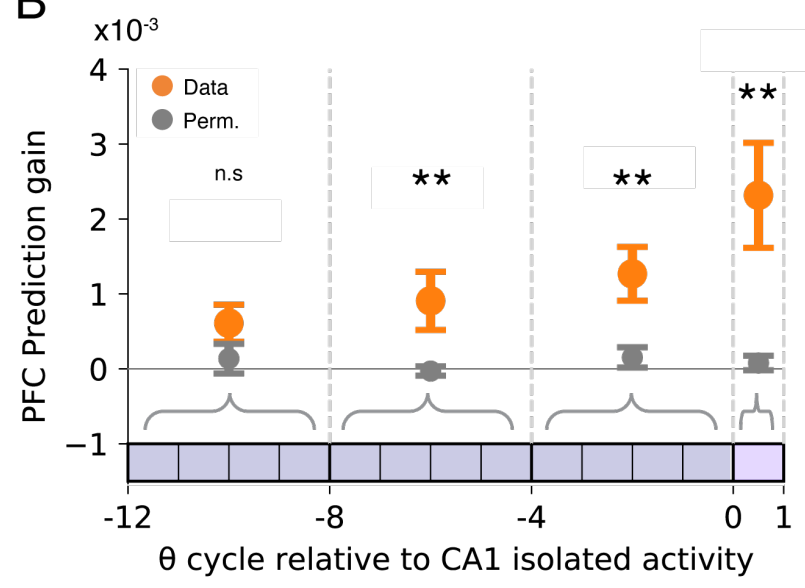

C

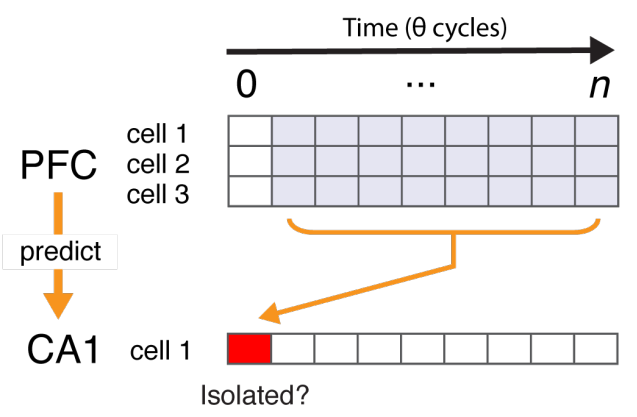

$\mathrm{D}$

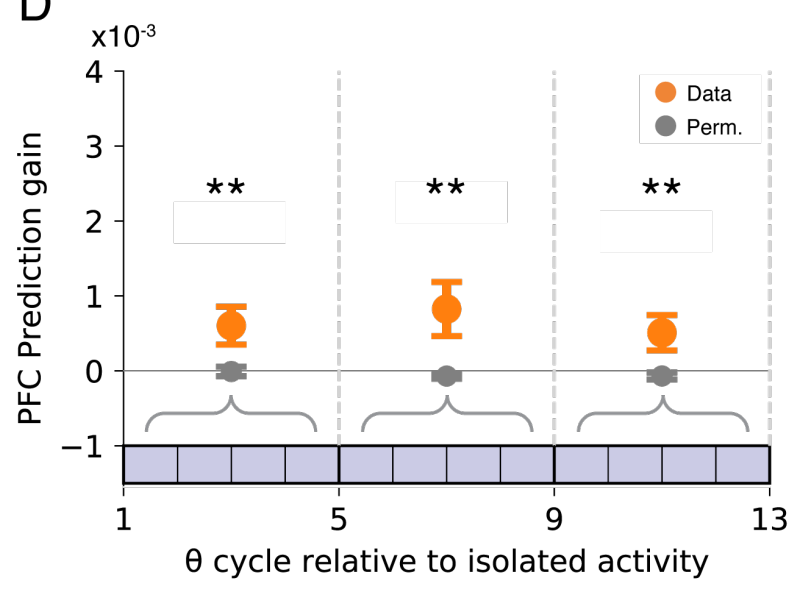

$410 \quad$ Figure 5.

411 A. PFC activity leading up to isolated spiking is used to predict the future occurrence of isolated spiking in one CA1 place cell.

413 B. Prediction gain (Mean \pm SEM) of GLMs where PFC spiking activity is used to predict whether an upcoming CA1 theta cycle contains isolated or non-isolated control spiking activity for a given CA1 place cell $(\mathrm{n}=158)$. Pairwise permutation test $(* * \mathrm{p}<0.05)$ for mean with multiple comparison correction: $\mathrm{p}=0.079, \mathrm{p}=0.0027, \mathrm{p}=0.0006$ and $\mathrm{p}<0.0002$ for each

417 group respectively.

C. PFC activity after isolated spiking is used to predict the previous occurrence of isolated spiking in one CA1 place cell.

420 D. Prediction gain (Mean \pm SEM) of GLMs where PFC spiking activity is used to predict 421 whether a previous CA1 theta cycle contained isolated or non-isolated control spiking 422 activity for a given CA1 place cell $(\mathrm{n}=162)$. Pairwise permutation test $(* * \mathrm{p}<0.05)$ for mean 
424 respectively.

425
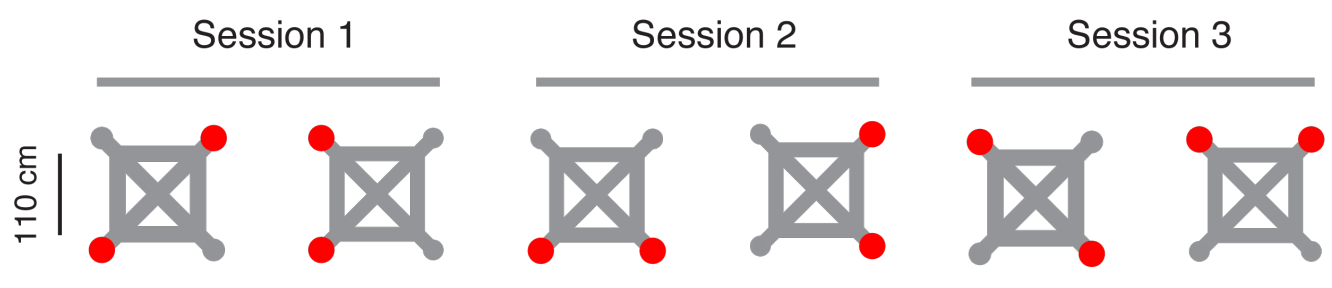

Figure S1. Task schematic.

The maze has 4 potential reward locations (wells) located on the corners of the maze but

429 only 2 will deliver reward for a given contingency. The animal needs to find the 2 wells that

430 deliver reward (red circles) and alternate between the two to continuously receive reward. All

431 reward wells are connected by paths (gray) and the rat is free to choose any path. The locations

432 of the rewarded wells change within session, between sessions and/or between days. The pairs of

433 wells that are rewarded vary across sessions and the rat needs to learn the locations of the

434 rewarded wells for every contingency by trial and error. 


\section{Adjacent activity}

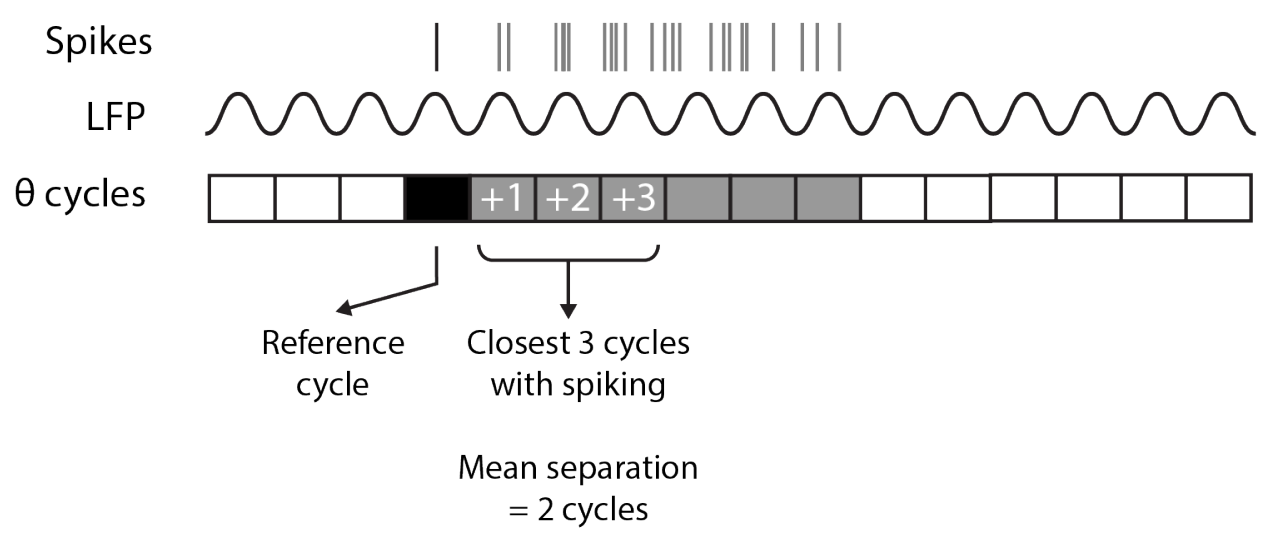

Isolated activity

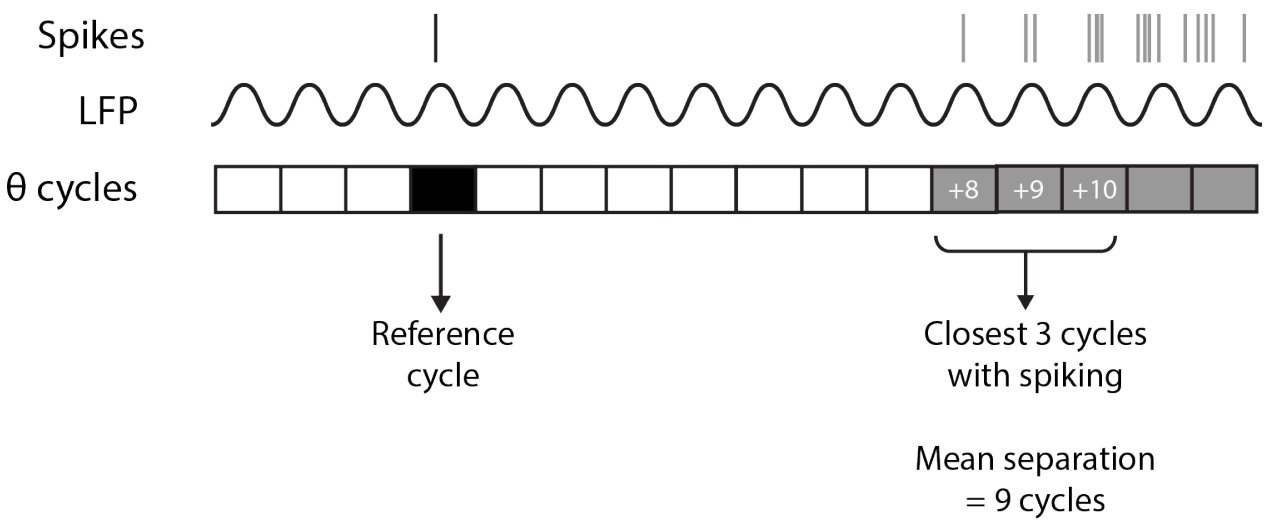

Figure S2. Adjacent and Isolated activity.

440 theta cycles with spiking. 


\section{A}

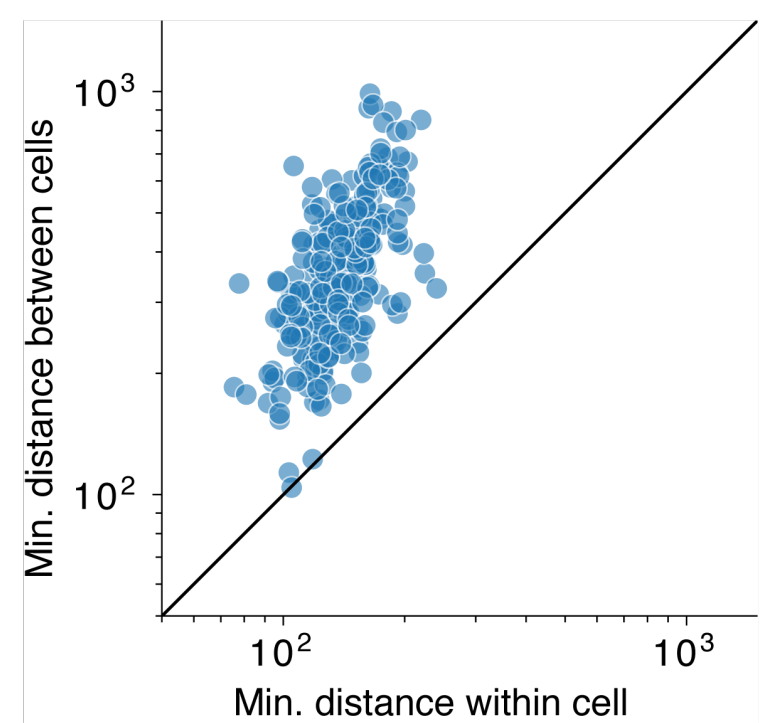

B

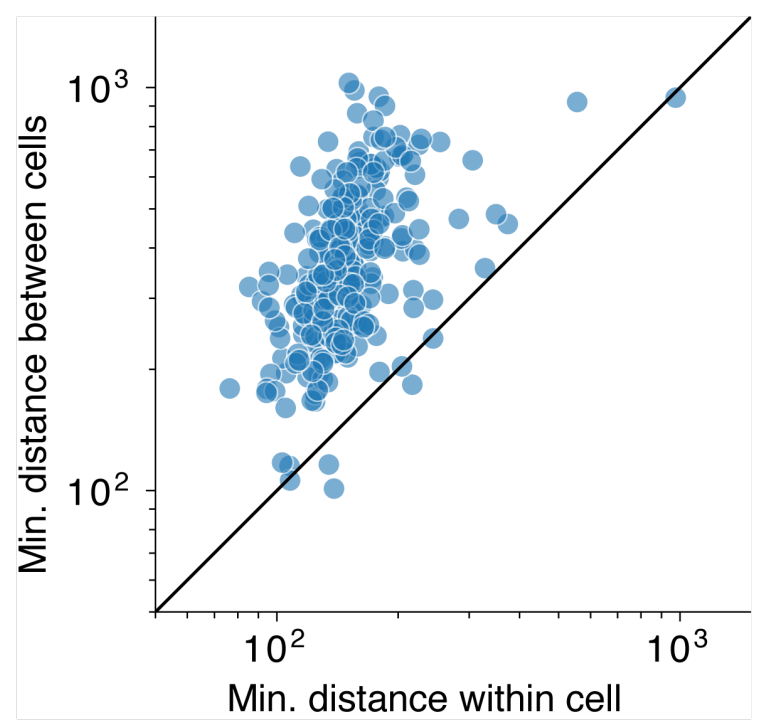

443 Figure S3. Isolated spiking activity is not due to spike assignment errors.

444 A. Minimum Euclidean distance between the spike waveform of adjacent activity spikes within

445 each cell versus all other place cell recorded on the same tetrode $(\mathrm{n}=260$ individual cells

446 compared to all other cells on the tetrode). Wilcoxon signed-rank test: $\mathrm{p}=2.9 \times 10^{-51}$.

447 B. Minimum Euclidean distance between the spike waveform of spikes classified as isolated

448 activity within each cell versus all other place cell recorded on the same tetrode $(n=260)$.

449 Wilcoxon signed-rank test: $\mathrm{p}=8.8 \times 10^{-51}$. 
A

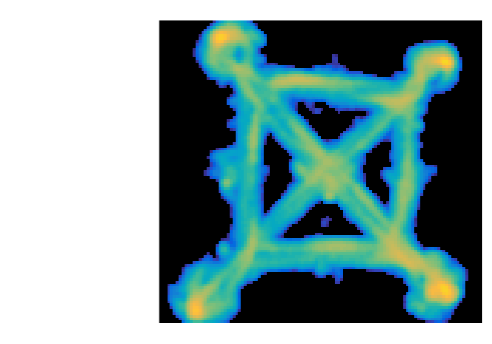

Adjacent
Isolated

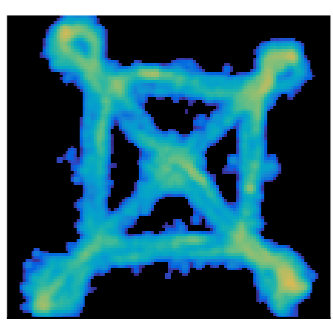

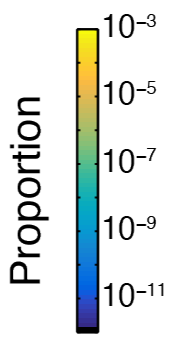

Difference

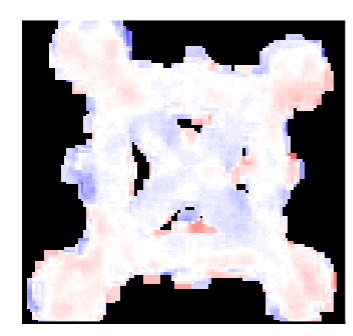

Z

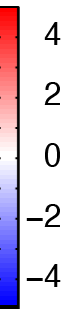

B

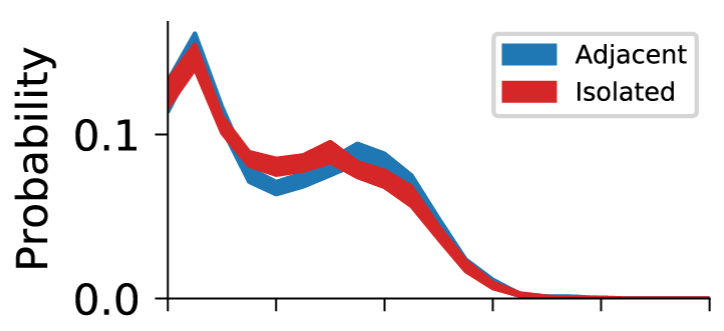

C
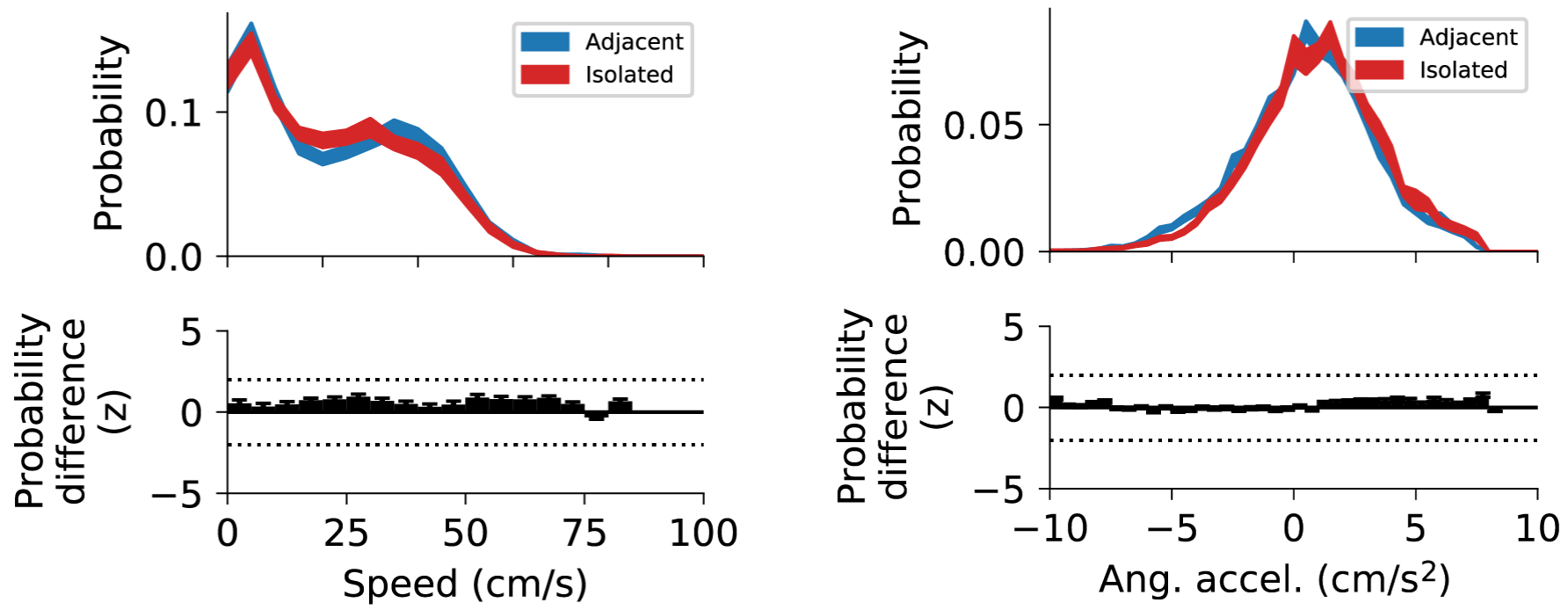

Figure S4. Animal location and movement correlates are similar for isolated and adjacent spiking.

A. Normalized spatial distribution of theta cycles with adjacent (left) or isolated (center) spiking. Normalized difference between the spatial distributions (right).

B. Distribution of animal speed (Mean \pm SEM) at the time of adjacent or isolated activity (top). Significance of the difference $(\mathrm{z})$ between the two distributions as determined using a permutation test (bottom). Dotted lines indicate $\pm 2 \mathrm{z}$.

C. Distribution of animal angular acceleration (Mean \pm SEM) at the time of adjacent or isolated activity (top). Significance of the difference (z) between the two distributions as determined 
A

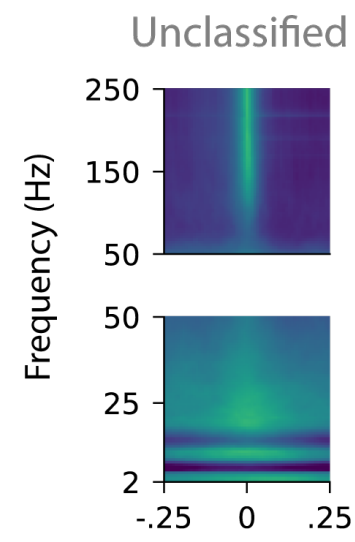

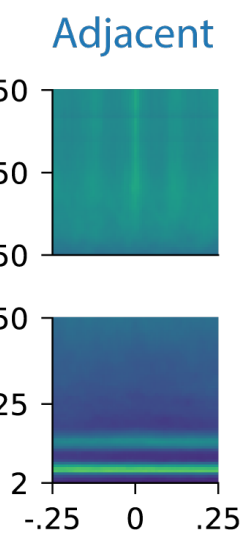

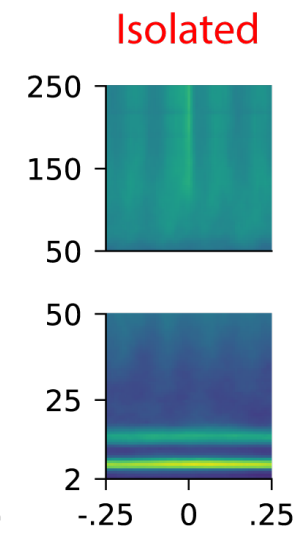

B

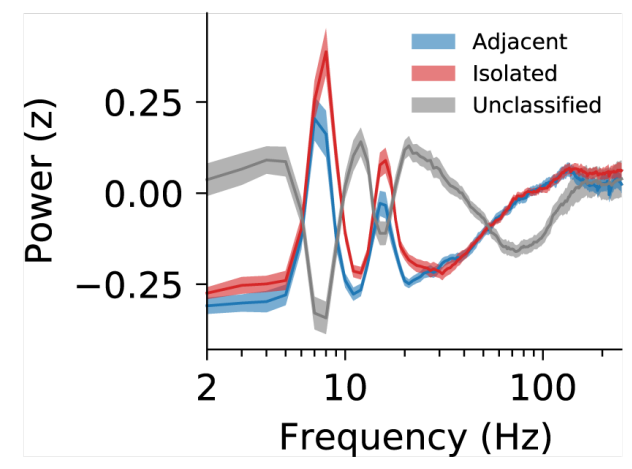

Time rel. to isolated activity (s)

$\begin{array}{ccc}-0.4 & 0 & 0.4 \\ & z & \end{array}$

\section{Figure S5. Network spectral signature around unclassified, adjacent and isolated spiking}

466 activity.

467 A. Mean spike triggered spectrogram for unclassified (left), adjacent (center), and isolated

468 (right) spiking activity ( $\mathrm{n}=170$ cells). Top panels show frequency ranges $50-250 \mathrm{~Hz}$. Bottom panels show frequency ranges $2-50 \mathrm{~Hz}$.

470

B. Mean spectral power for a $50 \mathrm{~ms}$ window centered at $0 \mathrm{~ms}$ lag (Median $\pm 95 \% \mathrm{CI}$ ). 
A
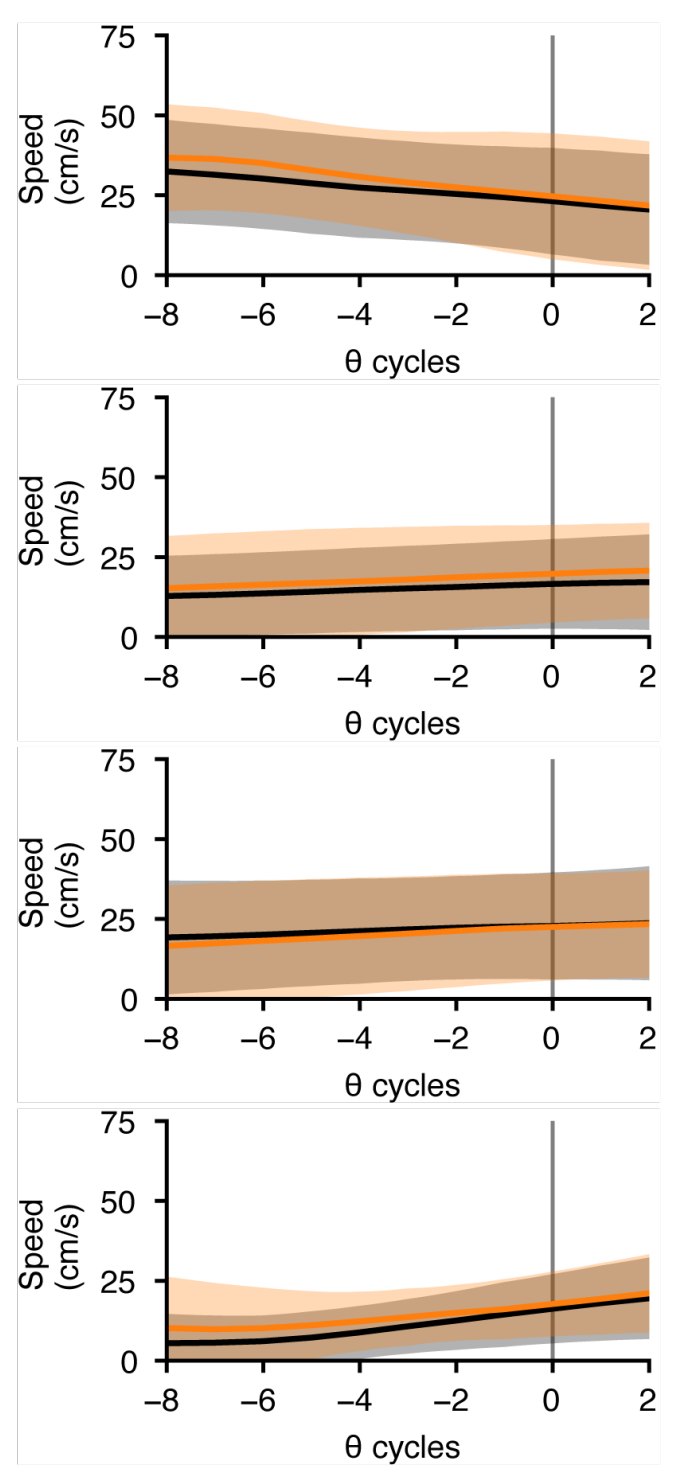

B

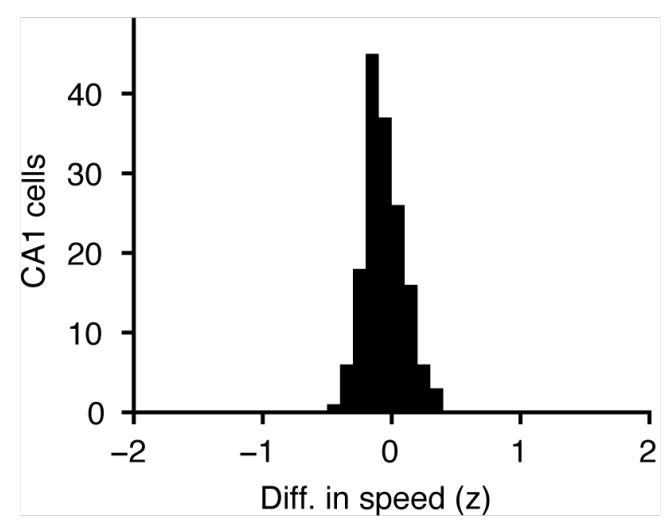

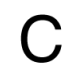

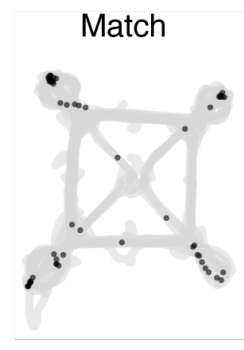

Match

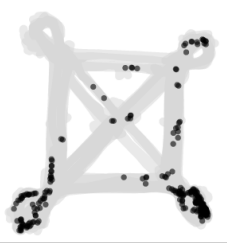

Match

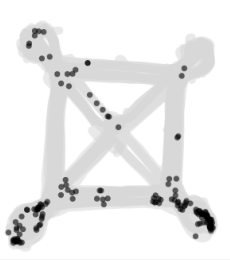

Match

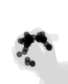

Isolated

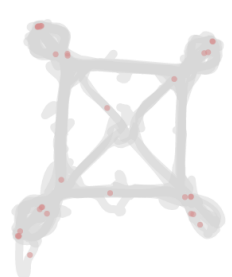

Isolated

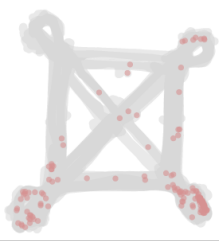

Isolated

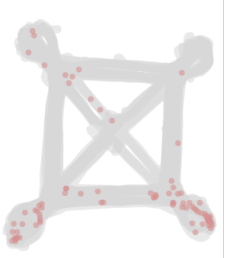

Isolated

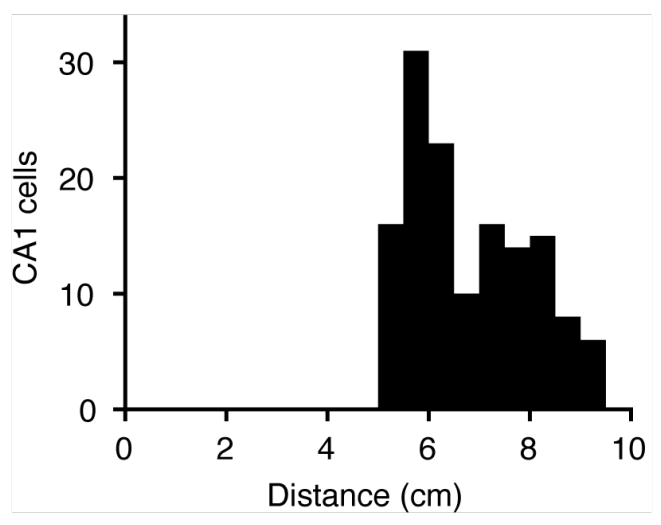




\section{Figure S6. Each theta cycle with isolated place cell activity is matched with non-isolated}

474 cycles for speed, trajectory and location.

475 A. Speed match profiles for examples in Figure 4B.

476 B. Distribution of mean difference in speed between matched and isolated cycles. Speed profile

477 of matched cycles were on average within -0.06 standard deviations of the speed profile of

478 the isolated cycles. The difference is expressed as a z-score normalized against the speed

479 distribution of isolated cycles.

480 C. Location match profiles examples in Figure 4B.

481 D. Distribution of the mean distance in $\mathrm{cm}$ between matched and isolated cycles. The location of 482 the animal on matched cycles was on average $7.5 \mathrm{~cm}$ from the location of the isolated cycle.

A

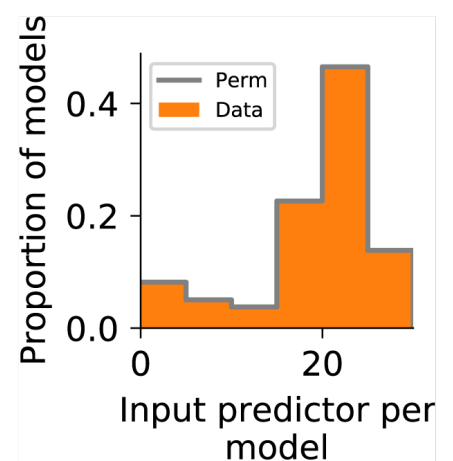

C

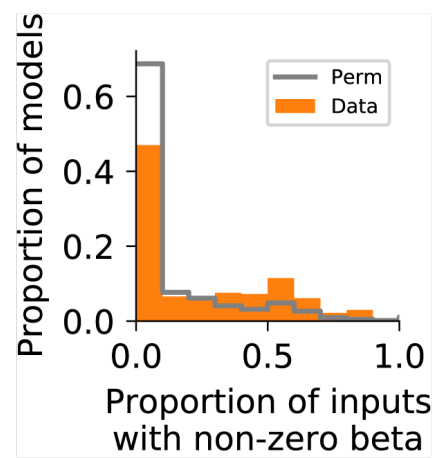

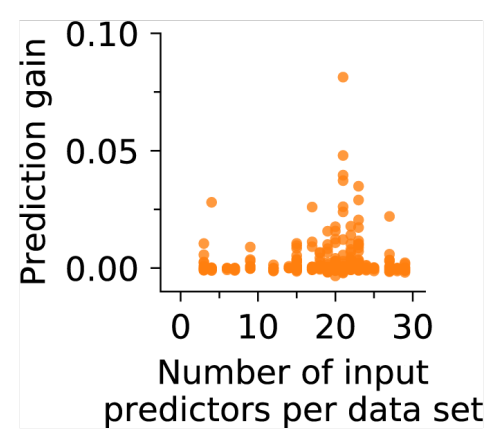

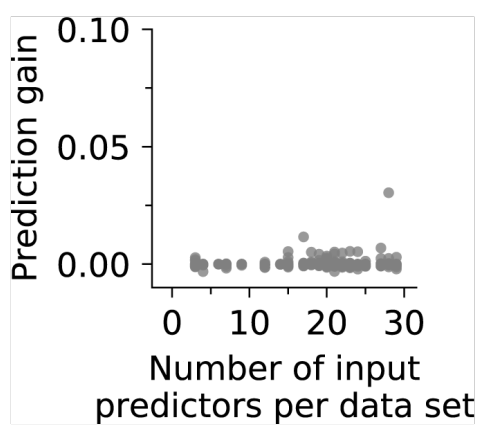

$\mathrm{D}$

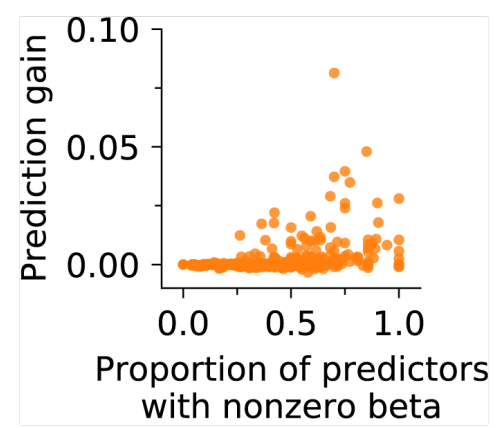

484

Figure S7. Model quality controls for GLMs using PFC activity to predict CA1 isolated activity.

487 A. Input predictor count for actual and permuted datasets. Wilcoxon rank-sum test $\mathrm{p}=1.0$. 
B. Prediction gain is not significantly correlated with the total number of input predictors used for prediction. Data: $\mathrm{R}^{2}=0.00121, \mathrm{p}=0.384$; Permutation control: $\mathrm{R}^{2}=0.00387, \mathrm{p}=0.118$.

C. Models using actual data have higher proportions of predictors with non-zero $\beta$ coefficients. Wilcoxon rank-sum test $\mathrm{p}=1.22 \times 10^{-16}$.

D. Prediction gain is positively correlated with the proportion of input predictors with non-zero beta coefficients. This is found in both actual (left) and permuted (right) datasets. Data: $\mathrm{R}^{2}=0.133, \mathrm{p}=3.10 \times 10^{-21}$; Permutation control: $\mathrm{R}^{2}=0.0537, \mathrm{p}=0.3 .80 \times 10^{-9}$. points and models are shown.

\section{Methods}

500 The data used in this study came from the same dataset used in previous publications (Yu, Kay et 501 al. 2017, Yu, Liu et al. 2018).

\section{Animal and behavior}

Experiments followed guidelines from the University of California San Francisco Institutional Animal Care and Use Committee and US National Institutes of Health. Six LongEvans rats (male, 500-700g, 4-9 months of age) were first trained to traverse a linear track (1m)

507 for reward (evaporated milk, Carnation brand, with 5\% added sucrose). Next, the animals were 508 trained on a foraging task 21 days after surgery (Yu, Kay et al. 2017, Yu, Liu et al. 2018).

509 Briefly, the task has four possible reward well locations, only two were chosen to deliver reward 510 at a given time. The rat is trained to visit the two rewarded location in alternation to receive 511 reward. The rewarded well locations changed within or between sessions, or between days. Two 512 to three session (15-45 minutes) were performed each day with interleaved rest sessions (20-60 513 minutes). Reward was delivered (100-300 $\mu \mathrm{l}$ at $20 \mathrm{ml} / \mathrm{min})$ using a syringe pump (NE-500

514 OEM, New Era Pump Systems Inc.) after the animal breaks an infrared beam at the well 515 location. 
The recording drive was 3D printed (PolyJetHD Blue, Stratasys Ltd.) and contained up to

51928 individually movable tetrodes. Tetrodes (Ni-Cr, California Fine Wire Company) were gold 520 plated to $250 \mathrm{kOhm}$ at $1 \mathrm{kHz}$.

521 The implanted recording drives targeted both dorsal CA1 (7 tetrodes) and dorsal PFC

522 (14-21 tetrodes, housed in one cannula angled at 20 degrees toward the midline). CA1 AP: -

$5233.8 \mathrm{~mm}$ and ML: 2.2mm. PFC (anterior cingulate cortex and dorsal prelimbic cortex): AP:

$524+2.2 \mathrm{~mm}, \mathrm{ML}+1.5 \mathrm{~mm}$ and $\mathrm{DV}$ between $1.88 \mathrm{~mm}$ to $2.72 \mathrm{~mm}$ depending on the AP and ML

525 coordinates of each tetrode.

526 Initially, tetrodes were adjusted to reach the target DV coordinate (PFC) or guided by

527 LFP and spiking patterns (CA1), every 2 days. Once the target was reached, tetrodes were

528 adjusted $(\sim 30 \mu \mathrm{m})$ to improve cell isolation at the end of an experiment day

\section{Histology}

Recording sites were marked with electrolytic lesions by passing current through each tetrode $(30 \mu \mathrm{A}, 3 \mathrm{~s})$ at the end of the experiment. Animals were perfused after 24 hours with

533 paraformaldehyde (4\% in PBS). The brain was removed, fixed (24 hours at room temperature),

534 cryoprotected $\left(30 \%\right.$ sucrose in PBS at $\left.4^{\circ} \mathrm{C}\right)$ and sectioned (coronal, $\left.50 \mu \mathrm{m}\right)$. Cresyl Violet was

535 used to stain the sections to identify sites of electrolytic lesions.

Recording

Data were recorded with the NSpike system (LMF and J. MacArthur, Harvard

539 Instrumentation Design Laboratory). Dim lighting was used during the experiment. An infrared

540 LED array was mounted on the headstage amplifier to for position tracking. Video was recorded

541 at $30 \mathrm{~Hz}$. We recorded LFP $(0.5-400 \mathrm{~Hz}$ at $1.5 \mathrm{kHz})$ and spiking activity $(600-6000 \mathrm{~Hz}$ or $300-$

$5426000 \mathrm{~Hz}$ at $30 \mathrm{kHz}$ ) from each tetrode channel. For spike detection referencing, a tetrode located

543 in corpus callosum was used for CA1 and a local tetrode without detected spikes was used for

544 PFC.

\section{Data preprocessing}

Manual spike clustering was performed based on peak amplitude, spike width and wave-

548 form principal components (MatClust, https://bitbucket.org/mkarlsso/matclust/src/master/). 
To reconstruct the position of the animal, the centroid of the front and back diodes from the LED array was automatically extracted from the video.

\section{Spike clustering quality}

To assess clustering quality, we analyzed the similarity in spike waveform within and across different units. We expect a well clustered unit to have spikes with waveforms that are similar to other spikes assigned to the same unit compared with spikes assigned to other units.

556 Potential spike misassignment can occur only for spiking events detected on the same tetrode.

557 We therefore compared spike waveforms from a given unit to spikes from all other units on the 558 same tetrode. We computed the Euclidean distance between the spike waveforms (4 channels)

559 for all pairs of spikes. Next, we compared the minimum waveform distance between spikes 560 belonging to the same unit, and between that unit and all other units. This was done separately 561 for spikes associated with isolated or adjacent theta cycles.

\section{Spatial spiking rate}

The occupancy-normalized rate was calculated by dividing the number of spikes by the occupancy of the animal per spatial bin $(2 \mathrm{~cm}$ by $2 \mathrm{~cm})$ in the environment. A 2-dimensional symmetric Gaussian kernel $(\sigma=2 \mathrm{~cm}$ and $12 \mathrm{~cm}$ spatial extent) was then used for smoothing.

\section{Theta cycle definition and classification}

The theta-frequency component of the raw LFP signal was extracted using an equiripple

570 finite impulse response band-pass filter $(6-12 \mathrm{~Hz})$. Given theta is associated with movement

571 states (Vanderwolf 1969), we used two criteria to exclude activity associated with immobility

572 periods. First, we exclude periods when the speed of the animal was less than $2 \mathrm{~cm} / \mathrm{s}$. Second, we

573 excluded periods with Sharp Wave/Ripples, which occur during immobility or periods of slow

574 movement, using previously described methods (Yu, Kay et al. 2017, Yu, Liu et al. 2018). For

575 SWR detection, we used a speed threshold of $<4 \mathrm{~cm} / \mathrm{s}$ to ensure SWRs occurring during

576 intermediate speeds $(>2 \mathrm{~cm} / \mathrm{s}$ but $<4 \mathrm{~cm} / \mathrm{s})$ are excluded. Spikes occurring during these excluded

577 periods are classed as "Excluded".

$578 \quad$ For spiking during the included periods, we classified each theta cycle and spikes

579 belonging to that cycle as "isolated" or "adjacent" activity. This was done per place cell. The 
classification was based on the mean number of cycles separating a given cycle with spiking to its nearest 3 other neighboring cycles with spiking. A mean separation of 8 cycles is the threshold for classification as adjacent as opposed to isolated. This is based on the distribution of cycle separation across the entire place cell population.

\section{Theta cycle spatial distribution}

To determine if isolated spiking occurred more frequently at certain locations in the environment compared with adjacent spiking, we first plotted the normalized spatial distribution of theta cycles containing each type of activity for each cell, averaged across the population. The spatial distribution of isolated and adjacent theta cycles for each place cell was calculated using the spatial spiking rate method described above, except with their respective cycles instead of spikes. To determine whether there are areas in the environment where the occurrence of adjacent and isolated activity differ, we applied a permutation technique. This involves first permuting the identity of each theta cycle labeled as having adjacent or isolated activity. The spatial distribution of the two permuted sets were calculated and subtracted from each other to obtain the difference. This was done 500 times to generate an expected distribution of differences. The actual difference in spatial distribution between isolated and adjacent activity was compared to the expected distribution and a z-score was calculated.

\section{Theta cycle movement correlates}

To determine whether isolated and adjacent activity were associated with distinct movement correlates, we compared the distribution of animal speed and angular velocity at times

602 of theta cycles containing each type of activity. This was done for each place cell and then 603 averaged across the population. To determine if the two distributions were significantly different, 604 we used a permutation approach. For each place cell, the identity of the theta cycle, whether it 605 contains isolated or adjacent, was permuted. The difference between the two distributions 606 permuted data sets was the recalculated. This was repeated 1000 times to obtain a distribution of 607 expected differences. The actual difference was expressed as a z-score relative to the expected 608 distribution. 
The theta phase of each spike is relative to the phase of the reference signal obtained

612 from the tetrode located in the corpus callosum. The mean phase preference for spiking activity

613 for each place cell is the circular mean of the phases of all spike. Theta phase concentration is the

614 magnitude of the vector sum of all spikes, where each spike is a unit vector with angle

615 corresponding to the phase of theta.

\section{Time aligned spectrogram}

To compute the spectral properties of network activity around spiking events we first used a bank of bandpass filters (center frequency $\pm 1 \mathrm{~Hz}$ ) to filter the LFP signal across the

620 frequency range $2-250 \mathrm{~Hz}$. Each filtered signal normalized by subtracting the mean and dividing

621 by the standard deviation. For each place cell, we then selected the normalized signal in a 500ms

622 window centered on the time of each spiking event and averaged across spikes. This was

623 repeated for spikes classified as belonging to isolated, adjacent theta cycles or excluded from

624 analysis (see Theta cycle definition and classification). We ensured equal number of spikes

625 were used to generate the average across spike types for each cell by sampling without

626 replacement to match the type with the lowest count. The average for each place cell was then

627 used to generate the mean for the entire population.

\section{Spiking coactivity}

630 We quantified the likelihood a pair of place cells having isolated activity in the same 631 theta cycle relative to the expected probability, similar to what has been done for SWRs (Cheng 632 and Frank 2008, Singer and Frank 2009, Yu, Kay et al. 2017). The expected probability is the

633 frequency of observing spiking from two cells in the same theta cycle given their relative

634 frequency of spiking. For each cell, its spike count during a theta cycle with isolated activity was

635 first binarized, where the cell was either spiking or not spiking in that theta cycle. The proportion 636 of all theta cycles where both cells spiked was the observed coactivity. The expected coactivity 637 was calculated by permuting the participation of each cell across all theta cycles with isolated 638 activity. This was repeated 1000 times and to generate a distribution expected proportion of theta 639 cycles with both cells have isolate activity. The observed proportion was converted to a z-score by subtracting the mean and dividing by the standard deviation of the expected distribution. This 
641 method accounts for the differences in the number of theta cycles with isolated activity for each

642 cell in the pair.

643 To determine the temporal relationship between adjacent activity for a pair of place cells,

644 we computed the cross-correlation between theta cycles with adjacent activity for a given cell

645 pair. First, we assigned each theta cycle of a given cell as having adjacent spiking or not. Then,

646 we cross-correlated the assignment for a pair of place cells, where the lag is measured in the

647 number of cycles. We then found the absolute lag with the maximum cross-correlation value

648 with for each place cell pair.

\section{Cycle matching}

For each place cell, we matched each theta cycle with isolated activity with control theta cycles without spiking. These control cycles were drawn from other task trials from the same session and matched as closely as possible for trajectory, speed, and location. Two control cycles

654 were selected for each actual cycle. Trajectory matching only included task trials where the animal performed the same trajectory and ensured the same direction of travel across all matched cycles. The speed matching process started with generating a reference speed profile distribution for a time interval around a theta cycle with isolated activity for a given cell. For each theta cycle 658 with isolated activity, we then chose two candidate theta cycles without spiking. The speed profile for each candidate cycle around the same interval was compared with the reference distribution. The candidate cycle was accepted if the mean speed deviation compared with the reference distribution is $<1 \sigma$ The next inclusion criteria for the candidate cycle was having a location $<10 \mathrm{~cm}$ from the theta cycle with isolated activity. This selection process was done

663 without replacement. Only place cells with greater than 100 input cycles, including both isolated 664 and matched cycles, were included in the analysis.

\section{Spiking normalization to theta cycles}

For illustration purposes in Figure 4A, we converted PFC and CA1 spiking times to hippocampal theta cycle phases. Spiking times were transformed using linear interpolation from

669 time to theta phase relative to the start of the theta cycle with isolated activity. The mean spiking 670 rate was calculated with respect to theta cycles. 


\section{Spiking rate comparison}

673 We asked whether PFC spiking rate leading up to and including theta cycle with isolated

674 compared with matched control cycles. For each CA1 cell, we first identified cycles with isolated

675 spiking and control cycles without isolated spiking (see above). We next found all PFC cells that

676 were simultaneously recoded with the CA1 cell. For each of these PFC cells, we compared the

677 spike count in time intervals leading up to the theta cycle with or without isolated spiking from

678 the CA1 cell. Under the null hypothesis, the difference between the two sets of spike counts will

679 not be significantly different than chance. To estimate the significance of the spike count

680 difference, we used a permutation test where we permuted the theta cycle identity 1000 times

681 and calculated the difference between the PFC spiking for each permutation. The actual

682 difference was expressed as a z-score relative to this permuted distribution by subtracting the

683 mean and standard deviation of the permuted distribution. As an additional control to estimate

684 the expected difference between the groups, we repeated the analysis by first generating a

685 permuted data set where the theta cycle identity (with or without isolated spiking) was permuted.

686 This difference in the spiking rate of this permuted data set, expressed as a z-score, was

687 calculated as the actual data set.

\section{Generalized Linear Models}

We asked whether spiking activity from simultaneously recorded PFC cells can predict the occurrence of isolated activity from a CA1 cell. We built cross-validated Generalized Linear Models (GLMs) (Binomial distribution with logit link function) with elastic-net regularization, which combined LASSO and Ridge regularization to reduce overfitting (Zou and Hastie 2005).

694 To do this, we first identified theta cycles with isolate spiking for a CA1 cell. We next identified 695 another control set of theta cycles when the CA1 cell did not spike. These control cycles were matched for animal speed, movement direction and location (see Cycle matching). We created a

697 model for each CA1 cell to determine whether PFC spiking activity can distinguish between

698 cycles with or without isolated spiking in a time window relative to the cycle with isolated spiking. We first modelled using activity in the 12 cycles previous to the cycle with isolated activity and then the 12 cycles after the cycle with isolated activity. We ensured no other isolated

701 activity occurred in this window used for prediction. A 4-cycle bin size was used for grouping 


\section{Modelling parameters}

MATLAB's lassoglm function was used ('distr'='binomial', 'Link'='logit'). The

optimization was equally weighed between LASSO and Ridge methods ('alpha'=0.5). Shrinkage parameter $(\lambda)$ optimization was done using 3-fold cross-validation ('CV'=3) with 5 Monte Carlo repetitions ('MCReps'=5). We used 5-fold cross validation and averaged the outcome across the 5 cross-validations.

\section{Prediction gain}

Prediction gain describes the whether the models can predict the outcome above chance.

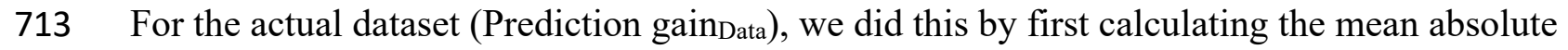

714 error (MAE) between the predicted and actual outcome for the validation partition (MAEData). To

715 estimate chance performance, we repeated the prediction 5000 times, each time with the outcome

716 permuted, and calculated the MAE. The chance MAE is the mean MAE of the 5000 control

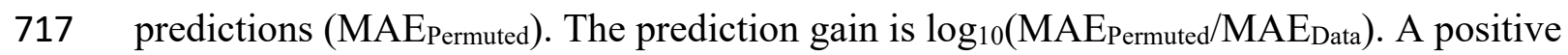

718 prediction gain means the Data group had a smaller error, or better prediction, compared with the

719 Permutated group (Rothschild, Eban et al. 2017).

We also used a second approach to estimate chance prediction. Instead of building the

721 model using actual data, we permuted the trial identity of PFC input, which preserves the input

722 spiking distribution but destroys any potential relationships between trials and the outcome in

723 CA1. We repeated the entire modelling procedure using permuted data and calculated the

724 prediction gain (Prediction gain Permuted).

To estimate whether there is significant above chance prediction of CA1 isolated activity

726 from PFC activity, we performed a permutation test ( $\mathrm{n}=10000$ permutations) on the mean

727 prediction gain between the actual (Prediction gain ${ }_{\text {Data }}$ ) and permuted (Prediction gain Permuted)

728 datasets. The Bonferroni correction was used to adjust the significance of the prediction to

729 account for multiple comparisons between time windows.

\section{PFC predictive ensemble correlation}

To determine whether there is specificity in the coordination between PFC and CA1

733 around the time of isolated activity, we asked if isolated activity for each CA1 cell was predicted 
734 by activity from distinct combination of PFC cells. This was done by calculating the Pearson's

735 correlation between the $\beta$ coefficients of CA1 models that were generated from data recorded on

736 the same day. We selected models that yielded a prediction gain $>1$, had a minimum of 5

737 predictors and had at least 2 CA1 models from the same day. This produced a dataset from 17

738 days, with a median of 3 (Q1: 2, Q3: 5) models per day, with 21 (Q1: 19, Q3: 23) predictors per

739 model, and with a median prediction gain of 1.014 (Q1: 1.0015, Q3: 1.026).

\section{Model quality assessment}

We checked the quality of our models by examining the relationship between prediction

743 gain and the contribution of predictors to the model. For these linear models, we used the value

744 of the $\beta$ coefficient to indicate the contribution of a predictor to the prediction, where predictors

745 with non-zero $\beta$ coefficient may contribute to the prediction. We examined how the prediction

746 value varied with the proportion of predictors with non-zero $\beta$ coefficients, or the total number of

747 input predictors, using linear regression. We also compared these relationships between models

748 with actual data or permuted data. Models with greater predictive power are expected to have

749 higher proportion of predictive features whereas the number of input predictors should not affect

750 the outcome.

751

752 Statistical Analyses

754 Circular statistical analyses were performed using the Circular Statistics Toolbox in MATLAB

755 (Berens 2009). Statistical tests were performed using standard MATLAB modules and Scipy

756 Statistical Functions (scipy.stats). All tests were two-sided. 


\section{Referenes}

765 Benchenane, K., A. Peyrache, M. Khamassi, P. L. Tierney, Y. Gioanni, F. P. Battaglia and S. I.

766 Wiener (2010). "Coherent theta oscillations and reorganization of spike timing in the

767 hippocampal- prefrontal network upon learning." Neuron 66(6): 921-936.

768 Berens, P. (2009). "CircStat: A MATLAB Toolbox for Circular Statistics." 2009 31(10): 21.

769 Brown, E. N., L. M. Frank, D. Tang, M. C. Quirk and M. A. Wilson (1998). "A statistical 770 paradigm for neural spike train decoding applied to position prediction from ensemble firing 771 patterns of rat hippocampal place cells." J Neurosci 18(18): 7411-7425.

772 Buckner, R. L., S. E. Petersen, J. G. Ojemann, F. M. Miezin, L. R. Squire and M. E. Raichle 773 (1995). "Functional anatomical studies of explicit and implicit memory retrieval tasks." J

$774 \quad$ Neurosci 15(1 Pt 1): 12-29.

775 Buzsaki, G. (2002). "Theta oscillations in the hippocampus." Neuron 33(3): 325-340.

776 Buzsáki, G. (2015). "Hippocampal sharp wave-ripple: A cognitive biomarker for episodic 777 memory and planning." Hippocampus 25(10): 1073-1188.

778

779

780

781

782

783

784

785

786

787

788

789

790

791

792

793
Buzsaki, G., L. W. Leung and C. H. Vanderwolf (1983). "Cellular bases of hippocampal EEG in the behaving rat." Brain Res 287(2): 139-171.

Cheng, S. and L. M. Frank (2008). "New Experiences Enhance Coordinated Neural Activity in the Hippocampus." Neuron 57(2): 303-313.

Eichenbaum, H. (2017). "Memory: Organization and Control." Annu Rev Psychol 68: 19-45.

Epsztein, J., M. Brecht and A. K. Lee (2011). "Intracellular determinants of hippocampal CA1 place and silent cell activity in a novel environment." Neuron 70(1): 109-120.

Faisal, A. A., L. P. Selen and D. M. Wolpert (2008). "Noise in the nervous system." Nat Rev Neurosci 9(4): 292-303.

Frank, L. M., E. N. Brown and M. Wilson (2000). "Trajectory Encoding in the Hippocampus and Entorhinal Cortex." Neuron 27(1): 169-178.

Guise, K. G. and M. L. Shapiro (2017). "Medial Prefrontal Cortex Reduces Memory Interference by Modifying Hippocampal Encoding." Neuron 94(1): 183-192 e188.

Hartline, H. K. (1938). "THE RESPONSE OF SINGLE OPTIC NERVE FIBERS OF THE VERTEBRATE EYE TO ILLUMINATION OF THE RETINA." American Journal of Physiology-Legacy Content 121(2): 400-415. 
Hill, A. J. and P. J. Best (1981). "Effects of deafness and blindness on the spatial correlates of hippocampal unit activity in the rat." Exp Neurol 74(1): 204-217. hippocampal place cell firing patterns." J Neurosci 33(8): 3443-3451.

Hyman, J. M., E. A. Zilli, A. M. Paley and M. E. Hasselmo (2005). "Medial prefrontal cortex cells show dynamic modulation with the hippocampal theta rhythm dependent on behavior." Hippocampus 15(6): 739-749.

801 Jadhav, S. P., G. Rothschild, D. K. Roumis and L. M. Frank (2016). "Coordinated Excitation and 802 Inhibition of Prefrontal Ensembles during Awake Hippocampal Sharp-Wave Ripple Events."

803 Neuron 90(1): 113-127.

804 Jay, T. M., J. Glowinski and A. M. Thierry (1989). "Selectivity of the hippocampal projection to 805 the prelimbic area of the prefrontal cortex in the rat." Brain research 505(2): 337-340.

806 Jensen, O. and J. E. Lisman (2000). "Position reconstruction from an ensemble of hippocampal 807 place cells: contribution of theta phase coding." J Neurophysiol 83(5): 2602-2609. structure within the noise." Trends Cogn Sci 13(2): 55-64.

810 Johnson, A. and A. D. Redish (2007). "Neural ensembles in CA3 transiently encode paths

811 forward of the animal at a decision point." J Neurosci 27(45): 12176-12189.

812 Jones, M. W. and M. A. Wilson (2005). "Theta Rhythms Coordinate Hippocampal-Prefrontal 813 Interactions in a Spatial Memory Task." PLoS Biology 3(12).

814 Karlsson, M. P. and L. M. Frank (2009). "Awake replay of remote experiences in the 815 hippocampus." Nature Neuroscience 12(7): 913-918.

816 Kay, K., J. E. Chung, M. Sosa, J. S. Schor, M. P. Karlsson, M. C. Larkin, D. F. Liu and L. M.

817 Frank (2020). "Constant Sub-second Cycling between Representations of Possible Futures in the 818 Hippocampus." Cell 180(3): 552-567 e525.

819 Kyd, R. J. and D. K. Bilkey (2003). "Prefrontal Cortex Lesions Modify the Spatial Properties of 820 Hippocampal Place Cells." Cerebral Cortex 13(5): 444-451.

821 Mainen, Z. F. and T. J. Sejnowski (1995). "Reliability of spike timing in neocortical neurons." 822 Science 268(5216): 1503-1506.

823 Masquelier, T. (2013). "Neural variability, or lack thereof." Front Comput Neurosci 7: 7. 
824 McNaughton, B. L., C. A. Barnes and J. O'Keefe (1983). "The contributions of position, 825 direction, and velocity to single unit activity in the hippocampus of freely-moving rats." Exp

826 Brain Res 52(1): 41-49.

827 Muller, R. U. and J. L. Kubie (1989). "The firing of hippocampal place cells predicts the future 828 position of freely moving rats." J Neurosci 9(12): 4101-4110.

829 Muller, R. U., J. L. Kubie and J. B. Ranck, Jr. (1987). "Spatial firing patterns of hippocampal

830 complex-spike cells in a fixed environment." J Neurosci 7(7): 1935-1950.

831 Murray, J. D., A. Bernacchia, D. J. Freedman, R. Romo, J. D. Wallis, X. Cai, C. Padoa-

832 Schioppa, T. Pasternak, H. Seo, D. Lee and X. J. Wang (2014). "A hierarchy of intrinsic

833 timescales across primate cortex." Nat Neurosci 17(12): 1661-1663.

834 Myroshnychenko, M., J. K. Seamans, A. G. Phillips and C. C. Lapish (2017). "Temporal

835 Dynamics of Hippocampal and Medial Prefrontal Cortex Interactions During the Delay Period of 836 a Working Memory-Guided Foraging Task." Cereb Cortex 27(11): 5331-5342.

837 O'Keefe, J. (1976). "Place units in the hippocampus of the freely moving rat." Exp Neurol 51(1): 838 78-109.

839 O'Keefe, J. and J. Dostrovsky (1971). "The hippocampus as a spatial map. Preliminary evidence 840 from unit activity in the freely-moving rat." Brain Research 34(1): 171-175.

841 O'Keefe, J. and M. L. Recce (1993). "Phase relationship between hippocampal place units and 842 the EEG theta rhythm." Hippocampus 3(3): 317-330.

843 Olton, D. S., M. Branch and P. J. Best (1978). "Spatial correlates of hippocampal unit activity." 844 Exp Neurol 58(3): 387-409.

845 Osborne, L. C., S. G. Lisberger and W. Bialek (2005). "A sensory source for motor variation." $846 \quad$ Nature 437(7057): 412-416.

847 Place, R., A. Farovik, M. Brockmann and H. Eichenbaum (2016). "Bidirectional prefrontal848 hippocampal interactions support context-guided memory." Nat Neurosci 19(8): 992-994.

849 Polyn, S. M., V. S. Natu, J. D. Cohen and K. A. Norman (2005). "Category-Specific Cortical

850 Activity Precedes Retrieval During Memory Search." Science 310(5756): 1963-1966.

851 Preston, A. R. and H. Eichenbaum (2013). "Interplay of hippocampus and prefrontal cortex in 852 memory." Curr Biol 23(17): R764-773.

853 Redish, A. D. (2016). "Vicarious trial and error." Nat Rev Neurosci 17(3): 147-159. 
Remondes, M. and M. A. Wilson (2015). "Slow- $\gamma$ Rhythms Coordinate Cingulate Cortical Responses to Hippocampal Sharp-Wave Ripples during Wakefulness." Cell reports 13(7): 1327-

8561335.

857

858

859

860

861

862

863

864

865

866

867

868

869

870

871

872

873

874

875

876

877

878

879

880

881

882

883

884 885

Rothschild, G., E. Eban and L. M. Frank (2017). "A cortical-hippocampal-cortical loop of information processing during memory consolidation." Nat Neurosci 20(2): 251-259.

Rugg, M. D. and K. L. Vilberg (2013). "Brain networks underlying episodic memory retrieval." Curr Opin Neurobiol 23(2): 255-260.

Sanders, H., C. Renno-Costa, M. Idiart and J. Lisman (2015). "Grid Cells and Place Cells: An Integrated View of their Navigational and Memory Function." Trends Neurosci 38(12): 763-775.

Schacter, D. L., D. Addis and R. L. Buckner (2007). "Remembering the past to imagine the future: the prospective brain." Nature Reviews Neuroscience 8(9): 657-661.

Schacter, D. L., N. M. Alpert, C. R. Savage, S. L. Rauch and M. S. Albert (1996). "Conscious recollection and the human hippocampal formation: evidence from positron emission tomography." Proc Natl Acad Sci U S A 93(1): 321-325.

Schedlbauer, A. M. and A. D. Ekstrom (2019). "Flexible network community organization during the encoding and retrieval of spatiotemporal episodic memories." Netw Neurosci 3(4): 1070-1093.

Shadlen, M. N. and W. T. Newsome (1998). "The variable discharge of cortical neurons: implications for connectivity, computation, and information coding." J Neurosci 18(10): 38703896.

Sherrington, C. S. (1906). "Observations on the scratch-reflex in the spinal dog." J Physiol 34(12): $1-50$.

Shin, J. D., W. Tang and S. P. Jadhav (2019). "Dynamics of Awake Hippocampal-Prefrontal Replay for Spatial Learning and Memory-Guided Decision Making." Neuron 104(6): 1110-1125 e1117.

Sigurdsson, T., K. L. Stark, M. Karayiorgou, J. A. Gogos and J. A. Gordon (2010). "Impaired hippocampal-prefrontal synchrony in a genetic mouse model of schizophrenia." Nature 464(7289): 763-767.

Simons, J. S. and H. J. Spiers (2003). "Prefrontal and medial temporal lobe interactions in longterm memory." Nature reviews. Neuroscience 4(8): 637-648.

Singer, A. C. and L. M. Frank (2009). "Rewarded Outcomes Enhance Reactivation of Experience in the Hippocampus." Neuron 64(6): 910-921. 
Skaggs, W. E., B. L. McNaughton, M. A. Wilson and C. A. Barnes (1996). "Theta phase precession in hippocampal neuronal populations and the compression of temporal sequences." Hippocampus 6(2): 149-172.

Spillmann, L. (2014). "Receptive fields of visual neurons: the early years." Perception 43(11): 1145-1176.

891 Squire, L. R., J. G. Ojemann, F. M. Miezin, S. E. Petersen, T. O. Videen and M. E. Raichle 892 (1992). "Activation of the hippocampus in normal humans: a functional anatomical study of memory." Proc Natl Acad Sci U S A 89(5): 1837-1841.

894 St Jacques, P. L., P. A. Kragel and D. C. Rubin (2011). "Dynamic neural networks supporting 895 memory retrieval." Neuroimage 57(2): 608-616.

896 Stein, R. B., E. R. Gossen and K. E. Jones (2005). "Neuronal variability: noise or part of the 897 signal?" Nat Rev Neurosci 6(5): 389-397.

898 Swanson, L. W. (1981). "A direct projection from Ammon's horn to prefrontal cortex in the rat." 899 Brain Research 217(1): 150-154.

900 Swanson, L. W. and W. M. Cowan (1977). "An autoradiographic study of the organization of the 901 efferent connections of the hippocampal formation in the rat." J Comp Neurol 172(1): 49-84.

902 Thompson, L. T. and P. J. Best (1989). "Place cells and silent cells in the hippocampus of freely903 behaving rats." J Neurosci 9(7): 2382-2390.

904 Tolhurst, D. J., J. A. Movshon and A. F. Dean (1983). "The statistical reliability of signals in 905 single neurons in cat and monkey visual cortex." Vision Res 23(8): 775-785.

906 Tomita, H., M. Ohbayashi, K. Nakahara, I. Hasegawa and Y. Miyashita (1999). "Top-down 907 signal from prefrontal cortex in executive control of memory retrieval." Nature 401(6754): 699908703.

909 Vanderwolf, C. H. (1969). "Hippocampal electrical activity and voluntary movement in the rat." 910 Electroencephalogr Clin Neurophysiol 26(4): 407-418.

911 Wang, D. V. and S. Ikemoto (2016). "Coordinated Interaction between Hippocampal Sharp912 Wave Ripples and Anterior Cingulate Unit Activity." J Neurosci 36(41): 10663-10672.

913 Wang, M., D. J. Foster and B. E. Pfeiffer (2020). "Alternating sequences of future and past 914 behavior encoded within hippocampal theta oscillations." Science 370(6513): 247-250.

915 Wilson, M. A. and B. L. McNaughton (1993). "Dynamics of the hippocampal ensemble code for 916 space." Science 261(5124): 1055-1058. 
917 Yu, J. Y. and L. M. Frank (2015). "Hippocampal-cortical interaction in decision making."

918 Neurobiol Learn Mem 117: 34-41.

919 Yu, J. Y., K. Kay, D. F. Liu, I. Grossrubatscher, A. Loback, M. Sosa, J. E. Chung, M. P.

920 Karlsson, M. C. Larkin and L. M. Frank (2017). "Distinct hippocampal-cortical memory

921 representations for experiences associated with movement versus immobility." Elife 6: e27621.

922 Yu, J. Y., D. F. Liu, A. Loback, I. Grossrubatscher and L. M. Frank (2018). "Specific

923 hippocampal representations are linked to generalized cortical representations in memory." $\underline{\text { Nat }}$

924 Commun 9(1): 2209.

925 Zhang, K., I. Ginzburg, B. L. McNaughton and T. J. Sejnowski (1998). "Interpreting neuronal 926 population activity by reconstruction: unified framework with application to hippocampal place 927 cells." J Neurophysiol 79(2): 1017-1044.

928 Zielinski, M. C., J. D. Shin and S. P. Jadhav (2019). "Coherent Coding of Spatial Position

929 Mediated by Theta Oscillations in the Hippocampus and Prefrontal Cortex." J Neurosci 39(23):

$930 \quad 4550-4565$.

931 Zou, H. and T. Hastie (2005). "Regularization and variable selection via the elastic net." Journal 932 of the Royal Statistical Society Series B-Statistical Methodology 67: 301-320. 